\title{
Thermal structure and cooling of neutron stars with magnetized envelopes
}

\author{
A. Y. Potekhin and D. G. Yakovlev \\ Ioffe Physico-Technical Institute, Politekhnicheskaya 26, 194021 St. Petersburg, Russia \\ Received 19 February 2001 / Accepted 17 April 2001

\begin{abstract}
The thermal structure of neutron stars with magnetized envelopes is studied using modern physics input. The relation between the internal $\left(T_{\mathrm{int}}\right)$ and local surface temperatures is calculated and fitted by analytic expressions for magnetic field strengths $B$ from 0 to $10^{16} \mathrm{G}$ and arbitrary inclination of the field lines to the surface. The luminosity of a neutron star with dipole magnetic field is calculated and fitted as a function of $B$, $T_{\text {int }}$, stellar mass and radius. In addition, we simulate cooling of neutron stars with magnetized envelopes. In particular, we analyse ultramagnetized envelopes of magnetars and also the effects of the magnetic field of the Vela pulsar on the determination of critical temperatures of neutron and proton superfluids in its core.
\end{abstract}

Key words. stars: neutron - dense matter - conduction - magnetic fields

\section{Introduction}

It is well known that theoretical models of cooling of isolated neutron stars (NSs) depend on the poorly known equation of state (EOS) of superdense matter in the NS interiors. Comparing calculated cooling curves (decrease of the NS effective surface temperature $T_{\mathrm{e}}$ in time) with observations gives a potentially powerful method of testing microscopic theories of superdense matter (e.g., Pethick 1992; Page 1997, 1998). Its practical implementation is restricted by the accuracy of observational determination of $T_{\mathrm{e}}$ and NS ages and by the quality of the physics input used in calculations. Great observational progress has been achieved recently after the launch of Chandra and Newton X-ray observatories. In the present paper we update theoretical models of thermal structure and evolution of NSs with magnetized envelopes.

Most of the observed NSs possess magnetic fields $B \sim$ $10^{12}-10^{13}$ G (e.g., Taylor et al. 1993). Some NSs are possibly magnetars, with $B>10^{14} \mathrm{G}$ (e.g., Thompson \& Duncan 1995; Kouveliotou et al. 1998, 1999; Mereghetti 2001). The internal NS magnetic field can be even higher. The strong magnetic field affects physical properties of all NS layers in many ways. For instance, the field $B \sim 10^{16} \mathrm{G}$ may affect the neutrino emissivity in the NS core (e.g., Baiko \& Yakovlev 1999). The field $B \gtrsim 10^{12} \mathrm{G}$ may change the electrical resistivity of NS cores, accelerating evolution of the internal field. In principle the thermal evolution may

Send offprint requests to: A. Y. Potekhin, e-mail: palex@astro.ioffe.rssi.ru be coupled to the magnetic one (e.g., Urpin \& Shalybkov 1995).

Thus modelling of the thermal structure and evolution of the magnetized NSs is a complicated task. In this paper we focus on two problems. First we consider the thermal structure of the outer magnetized NS envelope of density $\rho<\rho_{\mathrm{b}}=4 \times 10^{11} \mathrm{~g} \mathrm{~cm}^{-3}$ with the magnetic field $B \lesssim 10^{16} \mathrm{G}$. These envelopes produce thermal insulation (blanketing) of NS interiors. We solve this problem using updated physics input described in Sect. 2. The solution (Sect. 3) relates the internal NS temperature $T_{\text {int }}$ with the local effective surface temperature $T_{\mathrm{s}}$ and, therefore, with the integrated NS luminosity (or the mean effective surface temperature, $T_{\mathrm{e}}$ ). Second, in order to illustrate the obtained $T_{\mathrm{s}}\left(T_{\text {int }}\right)$ relation, we simulate (Sect. 4 ) cooling of NSs which possess a given dipole or radial magnetic field in the outer envelopes.

The effects of a strong magnetic field on thermodynamic and kinetic properties of the outer NS layers have been reviewed, for instance, by Yakovlev \& Kaminker (1994) and Ventura \& Potekhin (2001). The field affects the properties of all plasma components, the electron component usually being affected most strongly. Motion of free electrons perpendicular to the field lines is quantized in Landau orbitals with a characteristic transverse scale equal to the magnetic length $a_{\mathrm{m}}=(\hbar c / e B)^{1 / 2}=\lambda_{\mathrm{e}} / \sqrt{b}$, where $\lambda_{\mathrm{e}}=\hbar /\left(m_{\mathrm{e}} c\right)$ is the electron Compton wavelength, $b=\hbar \omega_{\mathrm{c}} / m_{\mathrm{e}} c^{2}=B_{12} / 44.14$ is the magnetic field strength expressed in relativistic units, $\omega_{\mathrm{c}}=e B / m_{\mathrm{e}} c$ is the electron cyclotron frequency, and $B_{12} \equiv B /\left(10^{12} \mathrm{G}\right)$. Except for the outermost parts of the NS envelopes, the electrons 
constitute degenerate, almost ideal gas. The electron entropy, magnetization, thermal and electrical conductivities, and other quantities exhibit quantum oscillations of the de Haas-van Alphen type. The oscillations occur under variations of density $\rho$ or $B$ whenever the electron Fermi momentum reaches the characteristic values $\sqrt{2 n b} m_{\mathrm{e}} c$ associated with occupation of Landau levels $n=1,2, \ldots$ These oscillations appreciably change the properties of degenerate electrons in the limit of a strongly quantizing field (e.g., Yakovlev \& Kaminker 1994) in which almost all electrons populate the ground Landau level. The latter case takes place at temperature $T \ll T_{\mathrm{B}}$ and density $\rho<\rho_{\mathrm{B}}$, where

$$
\begin{aligned}
& \rho_{\mathrm{B}}=m_{\mathrm{u}} n_{\mathrm{B}} A / Z \approx 7045 B_{12}^{3 / 2}(A / Z) \mathrm{g} \mathrm{cm}^{-3}, \\
& T_{\mathrm{B}}=\hbar \omega_{\mathrm{g}} / k_{\mathrm{B}} \approx 1.343 \times 10^{8}\left(B_{12} / \sqrt{1+x_{\mathrm{r}}^{2}}\right) \mathrm{K} .
\end{aligned}
$$

Here, $m_{\mathrm{u}}=1.66054 \times 10^{-24} \mathrm{~g}$ is the atomic mass unit, $A$ and $Z$ are the mean ion mass and charge numbers, $\omega_{\mathrm{g}}=$ $\omega_{\mathrm{c}} / \sqrt{1+x_{\mathrm{r}}^{2}}$ is the electron gyrofrequency,

$x_{\mathrm{r}}=\frac{\hbar\left(3 \pi^{2} n_{\mathrm{e}}\right)^{1 / 3}}{m_{\mathrm{e}} c} \approx 1.009\left(\rho_{6} Z / A\right)^{1 / 3}$

is the non-magnetic relativity parameter, $\rho_{6} \equiv$ $\rho /\left(10^{6} \mathrm{~g} \mathrm{~cm}^{-3}\right)$, and $n_{\mathrm{B}}=1 /\left(\pi^{2} \sqrt{2} a_{\mathrm{m}}^{3}\right)$ is the electron number density at which the Fermi energy reaches the first excited Landau level. In the case of a weakly quantizing magnetic field $\left(\rho \gtrsim \rho_{\mathrm{B}}, T \lesssim T_{\mathrm{B}}\right)$, the oscillations of electron quantities occur around the classical non-magnetic values and are typically not very pronounced. In the case of $T \gg T_{\mathrm{B}}$, the field can be treated as classical (non-quantizing) and the oscillations disappear.

The thermal structure of magnetized NS envelopes has been studied by a number of authors mainly using a plane-parallel approximation. More attention has been paid to the case of the radial magnetic field (normal to the surface). It has been thoroughly considered by Hernquist (1985), Van Riper (1988), Schaaf (1990a), Heyl \& Hernquist (1998b) for $B \lesssim 10^{14}$ G. In another paper Heyl \& Hernquist (1998a) analysed the case of higher fields, $B \sim 10^{15}-10^{16} \mathrm{G}$. One can consult the cited papers for references to earlier works. The principal conclusion of these studies is that the magnetic field reduces thermal insulation of the blanketing envelope by increasing the longitudinal (along the field lines) thermal conductivity of degenerate electrons due to Landau quantization of electron motion.

The thermal structure of the envelope with the magnetic field tangential to the surface has been analysed by Hernquist (1985), Schaaf (1990a), and Heyl \& Hernquist (1998a) for $B \lesssim 10^{14} \mathrm{G}$. In this case the field increases thermal insulation of the blanketing envelope due to the classical effect of reduction of the electron thermal conductivity transverse to the field because of the Larmor rotation.

The case of arbitrary inclination of the field to the surface was studied by Greenstein \& Hartke (1983) in the approximation of constant (density and temperature independent) longitudinal and transverse thermal conductivities. The authors proposed a very simple formula (Sect. 3.3) which relates the local surface and internal stellar temperatures, $T_{\mathrm{s}}$ and $T_{\mathrm{int}}$. It is constructed from two $T_{\mathrm{s}}\left(T_{\text {int }}\right)$ relations obtained for the radial and tangential magnetic fields. Page (1995) presented arguments that the formula of Greenstein \& Hartke is valid also for realistic, variable thermal conductivities. If so, one immediately gets the required $T_{\mathrm{s}}\left(T_{\mathrm{int}}\right)$ relation for any magnetic field inclination using the realistic relations for the radial and tangential fields. This method has been used by several authors (e.g., Shibanov \& Yakovlev 1996). Recently, the case of arbitrary field inclination has been studied also by Heyl \& Hernquist (1998b). In Sect. 3 we reconsider the thermal structure of the blanketing envelopes for any magnetic field inclination and compare our results with those of earlier studies.

Early simulations of cooling of magnetized NSs were performed assuming the radial magnetic field everywhere over the stellar surface (e.g., Nomoto \& Tsuruta 1987; Van Riper 1991). Since the radial magnetic field reduces the thermal insulation, these theories predicted acceleration of cooling of the magnetized NS accompanied by enhanced NS luminosity at the early (neutrino-dominated) cooling stage (at stellar ages $t \lesssim 10^{4}-10^{5} \mathrm{yr}$ ). Page (1995) and Shibanov \& Yakovlev (1996) simulated cooling of NSs with dipole magnetic fields. They showed that the decrease of thermal insulation of the stellar envelope near the magnetic equator partly compensated by its increase near the pole, and the magnetic field did not necessarily accelerate the cooling, in agreement with an earlier conjecture of Hernquist (1985). In a series of papers Heyl \& Hernquist (1997a, 1997b, 1998a, 1998b) proposed simplified models of cooling of magnetized NSs including the cases of ultrahigh surface magnetic fields, $B \sim 10^{15}-10^{16} \mathrm{G}$.

We illustrate our new models of magnetized NS envelopes with simulation of cooling of NSs (Sect. 4). We briefly discuss cooling of ultramagnetized NSs as well as cooling models of the Vela pulsar with the dipole magnetic field and superfluid core.

\section{Physics input}

\subsection{Equations of thermal structure and evolution}

The internal hydrostatic structure of a NS can be regarded as temperature-independent (e.g., Shapiro \& Teukolsky 1983). It is conventional (Gudmundsson et al. 1983) to separate the calculation of heat transport in the NS interior (at radius $r<R_{\mathrm{b}}$ ) and in the outer heat-blanketing envelope $\left(R_{\mathrm{b}} \leq r \leq R\right.$, where $R$ is the stellar radius). The choice of the boundary $R_{\mathrm{b}}$ must meet several requirements. The blanketing envelope should be thin $\left(R-R_{\mathrm{b}} \ll R\right)$ and contain negligibly small mass; there should be no large sources of energy generation or sink present there; it should serve as a good thermal blanket of the internal region; its thermal relaxation time should be shorter than time-scales of temperature variation in the 
internal region. In the studies of non-magnetized NSs, the boundary is usually taken at the density $\rho_{\mathrm{b}}=10^{10} \mathrm{~g} \mathrm{~cm}^{-3}$ (e.g., Gudmundsson et al. 1983; Potekhin et al. 1997 hereafter PCY). In the presence of a strong magnetic field we assume additionally that the temperature does not vary over the boundary $r=R_{\mathrm{b}}$ at any given moment. To make this requirement more realistic, we shift the boundary to the neutron drip density, $\rho_{\mathrm{b}}=4 \times 10^{11} \mathrm{~g} \mathrm{~cm}^{-3}$.

\subsubsection{Internal region}

In the internal region $\left(r<R_{\mathrm{b}}\right)$, magnetic fields $B \lesssim$ $10^{16} \mathrm{G}$ are either non-quantizing or weakly quantizing (degenerate electrons populate many Landau levels). For simplicity, we neglect the effects of magnetic fields in this region and use a spherically symmetric temperature distribution which obeys the classical equations of thermal evolution (Thorne 1977):

$$
\begin{aligned}
& \frac{\mathrm{e}^{-2 \Phi}}{4 \pi r^{2}} \sqrt{1-\frac{2 G m}{c^{2} r}} \frac{\partial}{\partial r}\left(\mathrm{e}^{2 \Phi} L\right)=-Q_{\nu}-C_{\mathrm{v}} \mathrm{e}^{-\Phi} \frac{\partial T}{\partial t}, \\
& \frac{L}{4 \pi r^{2}}=-\kappa \sqrt{1-\frac{2 G m}{c^{2} r}} \mathrm{e}^{-\Phi} \frac{\partial}{\partial r}\left(T \mathrm{e}^{\Phi}\right),
\end{aligned}
$$

where $Q_{\nu}(r)$ is the neutrino emissivity, $C_{\mathrm{v}}(r)$ is the heat capacity, $\kappa(r)$ is the thermal conductivity, $L(r)$ is the local luminosity defined as the non-neutrino heat flux transported through a sphere of radius $r$. Furthermore, $G$ is the gravitational constant, $m(r)$ is the gravitational mass inside the sphere of radius $r$, and $\Phi(r)$ is the metric function determined by the stellar model. Notice that at the stellar surface $\mathrm{e}^{\Phi(R)}=\left(1-r_{\mathrm{g}} / R\right)^{-1 / 2}$, where $r_{\mathrm{g}}=2 G M / c^{2}=$ $2.95\left(M / M_{\odot}\right) \mathrm{km}$ is the Schwarzschild radius defined by the total gravitational NS mass $m(R)=M$. On the righthand side of Eq. (4) we neglect the rate of heat production (e.g., by internal friction due to differential rotation, see Page 1997, 1998 and references therein). This effect is important for relatively old and cold NSs, which we will not consider here. To solve Eqs. (4) and (5), we use the computer code described by Gnedin et al. (2001, hereafter GYP). After thermal relaxation within the NS, at $t \gtrsim 100$ yr the redshifted temperature $\widetilde{T}(t) \equiv T(r, t) \mathrm{e}^{\Phi(r)}$ becomes constant throughout the internal region.

\subsubsection{Blanketing envelope}

The thermal structure of the blanketing envelope is studied in the stationary, local plane-parallel approximation to relate the local effective surface temperature $T_{\mathrm{s}}$ to the temperature $T_{\mathrm{int}}$ at the inner boundary of the envelope. Everywhere on the surface, except possibly in tiny regions where the field is almost tangential, one may assume that a scale of temperature variation over the surface is much larger than the thickness of the blanketing envelope. This leads to the one-dimensional approximation for the heat diffusion equation:

$F \equiv \sigma T_{\mathrm{s}}^{4}=\kappa \frac{\mathrm{d} T}{\mathrm{~d} z}=\frac{16 \sigma}{3} \frac{T^{3} \mathrm{~d} T}{\mathrm{~d} \tau}, \quad \kappa \equiv \frac{16 \sigma T^{3}}{3 K \rho}$, where $F$ is the local heat flux density (constant throughout a given local part of the envelope), $\kappa$ is an effective thermal conductivity along the normal to the surface, $\sigma$ is the Stefan-Boltzmann constant, $K$ is the mean opacity (Sect. 2.3), and $z=(R-r) \mathrm{e}^{\Phi(R)}$ is the local proper depth.

Integration of Eq. (6) gives a temperature profile $T \approx$ $T_{\mathrm{S}}\left(\frac{3}{4} \tau+\frac{1}{2}\right)^{1 / 4}$, where $\tau=\int_{-\infty}^{z} K \rho \mathrm{d} z$ is the optical depth, and the integration constant corresponds to the Eddington approximation $\left(\tau=\frac{2}{3}\right.$ at the radiative surface, where $T=$ $\left.T_{\mathrm{s}}\right)$. A more accurate boundary condition requires solution of the radiative transfer equation in the NS atmosphere (e.g., Shibanov et al. 1998).

At $z \ll R$ the general relativistic equation of hydrostatic equilibrium can be reduced to the Newtonian form: $\mathrm{d} P / \mathrm{d} z=g \rho$, where $g=G M /\left(R^{2} \sqrt{1-r_{\mathrm{g}} / R}\right)$ is the surface gravity. Together with Eq. (6), it leads to the thermal structure equation

$\frac{\mathrm{d} \log T}{\mathrm{~d} \log P}=\frac{3}{16} \frac{P K}{g} \frac{T_{\mathrm{s}}^{4}}{T^{4}}$

where $P$ is the pressure.

The thermal conductivity tensor of magnetized plasma is anisotropic. It is characterized by the conductivities parallel $\left(\kappa_{\|}\right)$and transverse $\left(\kappa_{\perp}\right)$ to the field, and by the offdiagonal (Hall) component. In the plane-parallel approximation Eq. (6) contains the effective thermal conductivity

$\kappa=\kappa_{\|} \cos ^{2} \theta+\kappa_{\perp} \sin ^{2} \theta$,

where $\theta$ is the angle between the field lines and the normal to the surface. In case $\theta=0$ the heat is transported solely by the parallel conductivity, $\kappa=\kappa_{\|}$, while in case $\theta=90^{\circ}$ it is transported by the transverse conductivity, $\kappa=\kappa_{\perp}$. These special cases will be referred to as the parallel $(\|)$ and transverse $(\perp)$ conduction cases. Actually, $B$ and $\theta$ vary slowly over the surface. The total stellar luminosity is

$L=\int F \mathrm{~d} \Sigma=\sigma \int T_{\mathrm{s}}^{4} \mathrm{~d} \Sigma \equiv 4 \pi \sigma R^{2} T_{\mathrm{e}}^{4}$,

where $\mathrm{d} \Sigma=R^{2} \mathrm{~d} \Omega$ is the surface element determined by corresponding solid angle $\mathrm{d} \Omega$, and $T_{\mathrm{e}}$ is the (mean) effective temperature to be distinguished from the local effective temperature $T_{\mathrm{s}}$. Naturally, $T_{\mathrm{e}}=T_{\mathrm{s}}$ for a non-magnetic NS.

Assuming the dipole field, we can use the generalrelativistic solution (Ginzburg \& Ozernoy 1964)

$$
\begin{aligned}
& B(\chi)=B_{\mathrm{p}} \sqrt{\cos ^{2} \chi+a^{2} \sin ^{2} \chi}, \quad \tan \theta=a \tan \chi \\
& a=-\frac{(1-x) \ln (1-x)+x-0.5 x^{2}}{\left[\ln (1-x)+x+0.5 x^{2}\right] \sqrt{1-x}}
\end{aligned}
$$

where $B_{\mathrm{p}}$ is the field strength at the magnetic pole, $\chi$ is the polar angle, and $x=r_{\mathrm{g}} / R$.

The quantities $T_{\mathrm{s}}, T_{\mathrm{e}}$ and $L$ refer to a local reference frame at the NS surface. The redshifted ("apparent") quantities as detected by a distant observer are 
(Thorne 1977): $T_{\mathrm{s}}^{\infty}=T_{\mathrm{s}} \sqrt{1-r_{\mathrm{g}} / R}, \quad T_{\mathrm{e}}^{\infty}=$ $T_{\mathrm{e}} \sqrt{1-r_{\mathrm{g}} / R}, L_{\infty}=\left(1-r_{\mathrm{g}} / R\right) L$.

Crucial for the thermal evolution is the relation between $T_{\mathrm{s}}$ and $T_{\mathrm{int}}=T\left(R_{\mathrm{b}}\right)$. For non-magnetic blanketing envelopes composed of iron, this relation was studied by Gudmundsson et al. (1983), while the non-magnetic envelopes of various chemical compositions were analysed by PCY. Ventura \& Potekhin (2001) presented an analytic analysis of the $T_{\mathrm{int}}\left(T_{\mathrm{s}}\right)$ relation for the envelopes without magnetic fields and with strong magnetic fields; Heyl \& Hernquist (1998a) performed a semianalytic investigation of the case of strong magnetic field. The results of Ventura \& Potekhin (2001) and Heyl \& Hernquist (1998a) are rather approximate because of a number of simplifying assumptions discussed by Ventura \& Potekhin (2001).

The validity of the one-dimensional approximation can be checked by direct two-dimensional simulation of the heat transport in the blanketing envelope. Such simulation has been attempted by Schaaf (1990b) for a homogeneously magnetized NS under many simplified assumptions, so that a more realistic study is required. The heat conduction from hotter to cooler zones along the surface or possible meridional and convective motions can smooth the temperature variations over the NS surface. Nevertheless, the one-dimensional approximation seems to be sufficient for our cooling calculations presented below.

\subsection{Equation of state}

In the inner region of a NS, the effects of magnetic field are assumed to be weak and we use the same EOSs as in GYP. Specifically, in the core, we use the moderately stiff phenomenological EOS of matter composed of neutrons, protons and electrons, as proposed by Prakash et al. (1988), in its simplified version suggested by Page \& Applegate (1992). In the inner envelope, we use the model of groundstate matter (Negele \& Vautherin 1973) and describe the properties of atomic nuclei by the smooth composition model (Kaminker et al. 1999). The core-crust interface is placed at $\rho=1.5 \times 10^{14} \mathrm{~g} \mathrm{~cm}^{-3}$.

The outer envelope is assumed to be composed of iron, which can be partially ionized at $\rho \lesssim 10^{6} \mathrm{~g} \mathrm{~cm}^{-3}$. Following PCY, we employ the mean-ion approximation and adjust an effective ion charge to a more elaborate EOS: the Opacity Library (OPAL) EOS (Rogers et al. 1996) at $B=0$ and the Thomas-Fermi EOS of Thorolfsson et al. (1998) at $B=\left(10^{10}-10^{13}\right) \mathrm{G}$. The tabular entries of these EOSs in $\rho$ and $T$ are interpolated in the same way as in PCY. When necessary, we interpolate the effective charge at intermediate $B$. Since no reliable EOS of iron has been published for $B>10^{13} \mathrm{G}$, we use the effective charge obtained at $B=10^{13} \mathrm{G}$ for higher $B$.

Thus, in our model of the outer envelope, the pressure is produced by magnetized Fermi gas of electrons and by the gas of classical ions with an effective charge. We neglect the anomalous magnetic moments of the nuclei, which is a good approximation at $B \ll 10^{16} \mathrm{G}$ (cf. Broderick et al. 2000; Suh \& Mathews 2001). The electron pressure can be expressed through the chemical potential $\mu$ and $T$ (e.g., Blandford \& Hernquist 1982):

$P_{\mathrm{e}}=P_{\mathrm{r}} \frac{b \tau_{0}^{3 / 2}}{\sqrt{2} \pi^{2}} \sum_{n=0}^{n_{\max }}\left(2-\delta_{n 0}\right)(1+2 b n)^{1 / 4} I_{1 / 2}\left(\chi_{n}, \tau_{n}\right)$

where

$\chi_{n}=\frac{\mu-m_{\mathrm{e}} c^{2} \sqrt{1+2 b n}}{k_{\mathrm{B}} T}, \quad \tau_{n}=\frac{1}{\sqrt{1+2 b n}} \frac{T}{T_{\mathrm{r}}}$

$P_{\mathrm{r}}=m_{\mathrm{e}} c^{2} / \lambda_{\mathrm{e}}^{3} \approx 1.4218 \times 10^{25} \mathrm{dyn}^{-2}$ and $T_{\mathrm{r}}=$ $m_{\mathrm{e}} c^{2} / k_{\mathrm{B}} \approx 5.930 \times 10^{9} \mathrm{~K}$ are the relativistic units of pressure and temperature, respectively. The standard FermiDirac integral $I_{1 / 2}$ is given by a fit presented in Chabrier $\&$ Potekhin (1998). The chemical potential $\mu$ is found at given $\rho, T$, and $B$ using an analytic fit and iterative procedure described by Potekhin \& Yakovlev (1996).

\subsection{Opacities}

The heat is carried through the NS envelope mainly by electrons at relatively high densities and by photons near the surface (e.g., Gudmundsson et al. 1983). In general, the two mechanisms work in parallel, hence

$\kappa=\kappa_{\mathrm{r}}+\kappa_{\mathrm{c}}, \quad K^{-1}=K_{\mathrm{r}}^{-1}+K_{\mathrm{c}}^{-1}$,

where $\kappa_{\mathrm{r}}, \kappa_{\mathrm{c}}$ and $K_{\mathrm{r}}, K_{\mathrm{c}}$ denote the radiative (r) and electron (c) components of the conductivity and opacity.

Typically, the radiative conduction dominates $\left(\kappa_{\mathrm{r}}>\kappa_{\mathrm{c}}\right)$ in the outermost non-degenerate layers of a NS, whereas the electron conduction dominates $\left(\kappa_{\mathrm{c}}>\kappa_{\mathrm{r}}\right)$ in deeper, moderately and strongly degenerate layers. The $T_{\mathrm{s}}\left(T_{\mathrm{int}}\right)$ relation depends mainly on the conductivities in the sensitivity strip on the $\rho-T$ plane (Gudmundsson et al. 1983) placed near the turning point, where $\kappa_{\mathrm{c}} \sim \kappa_{\mathrm{r}}$.

\subsubsection{Radiative opacities}

Radiative opacities of partially ionized iron in strong magnetic fields were studied by Rajagopal et al. (1997), but neither tables nor analytic fits required for NS modelling were published. Fortunately, we will see that the $T_{\mathrm{s}}\left(T_{\mathrm{int}}\right)$ relation for a not too cold NS is not noticeably changed if we replace the radiative opacity of partially ionized iron by that of fully ionized iron. Therefore we base our opacity model on the assumption of full ionization. In this case, the two principal contributions into the opacity come from free-free transitions in electron-ion collisions and from Thomson scattering of photons by free electrons.

At $B=0$, the second process is described by the wellknown Thomson cross section $\sigma_{\mathrm{T}}$ in the non-relativistic limit $T \ll T_{\mathrm{r}}$ (e.g., Berestetski1 et al. 1992). The corresponding opacity is

$K_{\mathrm{T}}=\frac{n_{\mathrm{e}} \sigma_{\mathrm{T}}}{\rho}=\frac{8 \pi}{3}\left(\frac{e^{2}}{m_{\mathrm{e}} c^{2}}\right)^{2} \frac{n_{\mathrm{e}}}{\rho}$. 
The free-free absorption coefficient at $B=0$ was calculated by Karzas \& Latter (1961) as a function of the electron velocity and photon frequency $\omega$. Hummer (1988) produced an accurate fit to its thermal average in the range $10^{-4} \leq \xi \leq 10^{1.5}$ and $10^{-3} \leq T_{\mathrm{Ry}}<10^{3}$, where

$T_{\mathrm{Ry}} \equiv \frac{2 \hbar^{2}}{m_{\mathrm{e}} Z^{2} e^{4}} k_{\mathrm{B}} T=\frac{T_{6}}{0.15789 Z^{2}}, \quad \xi \equiv \frac{\hbar \omega}{k_{\mathrm{B}} T}$.

The ratio of the Rosseland mean, $K_{\mathrm{ff}}$, calculated using that fit, to the Thomson opacity can be written as

$\frac{K_{\mathrm{ff}}}{K_{\mathrm{T}}}=\frac{Z \alpha_{\mathrm{f}}}{3 c_{7}}\left(\frac{2}{\pi}\right)^{3 / 2}\left(\frac{T_{\mathrm{r}}}{T}\right)^{7 / 2} x_{\mathrm{r}}^{3} \approx \frac{2 \times 10^{4}}{c_{7}} \frac{Z^{2}}{A} \frac{\rho}{T_{6}^{7 / 2}}$,

where $\rho$ is measured in $\mathrm{g} \mathrm{cm}^{-3}, \alpha_{\mathrm{f}}=e^{2} /(\hbar c) \approx \frac{1}{137}$,

$c_{7}=\frac{15}{4 \pi^{4}} \int_{0}^{\infty} \frac{\xi^{7} \mathrm{e}^{-\xi}}{\left(1-\mathrm{e}^{-\xi}\right)^{3} \Lambda_{\mathrm{ff}}(T, \xi)} \mathrm{d} \xi$,

$\Lambda_{\mathrm{ff}}=(\pi / \sqrt{3}) \bar{g}_{\mathrm{ff}}$ is the Coulomb logarithm, and $\bar{g}_{\mathrm{ff}}$ is the thermally averaged Gaunt factor. In the Born approximation (e.g., Silant'ev \& Yakovlev 1980), $\Lambda_{\mathrm{ff}}=\mathrm{e}^{\xi / 2} K_{0}(\xi / 2)$, where $K_{0}$ is a modified Bessel function; then $c_{7}=316.824$. Since $\mathrm{e}^{\xi / 2} K_{0}(\xi / 2) \approx \sqrt{\pi / \xi}$ at $\xi \rightarrow \infty$, we can obtain a reasonable approximation to $\bar{g}_{\mathrm{ff}}$ by extending the accurate fit of Hummer (1988) beyond its boundary $\xi=10^{1.5}$ in the power law form $\bar{g}_{\mathrm{ff}} \propto \xi^{-1 / 2}$. Then we can calculate the integral in Eq. (17). The result is fitted (within $0.6 \%$ ) by

$c_{7}=\frac{108.8+77.6 T_{\mathrm{Ry}}^{0.834}}{1+0.502 T_{\mathrm{Ry}}^{0.355}+0.245 T_{\mathrm{Ry}}^{0.834}}$.

If free-free transitions and scattering at $B=0$ are important simultaneously, the Rosseland mean of the sum of the two opacities can be presented as

$K_{\mathrm{r}}=\left(K_{\mathrm{ff}}+K_{\mathrm{T}}\right) A(f, T), \quad f \equiv K_{\mathrm{ff}} /\left(K_{\mathrm{ff}}+K_{\mathrm{T}}\right)$.

The non-additivity factor $A(f, T)$ has been calculated by Silant'ev \& Yakovlev (1980) in the Born approximation (in which case it does not depend on $T$ ). Beyond the Born approximation, we obtain a function weakly depending on $T$, which can be fitted (within 1\%) as

$A(f, T)=1+\frac{1.097+0.777 T_{\mathrm{Ry}}}{1+0.536 T_{\mathrm{Ry}}} f^{0.617}(1-f)^{0.77}$.

Thus we can propose a simplified analytic treatment of the radiative opacity at $B=0$ as the opacity produced by freefree transitions and Thomson scattering alone. The effect of this simplified treatment is illustrated in Fig. 1. Here, we have applied the code for solving the thermal structure equation, described in PCY, with the updated conductive opacities (Potekhin et al. 1999) and two different sets of radiative opacities. The solid line on the upper panel is obtained using the OPAL radiative opacities (Iglesias \& Rogers 1996), whereas the dashed line is obtained using Eqs. (14)-(20). The resulting difference (lower panel) does not exceed several percent in the most important range of $T_{\mathrm{int}}$ shown in the figure. The difference between the

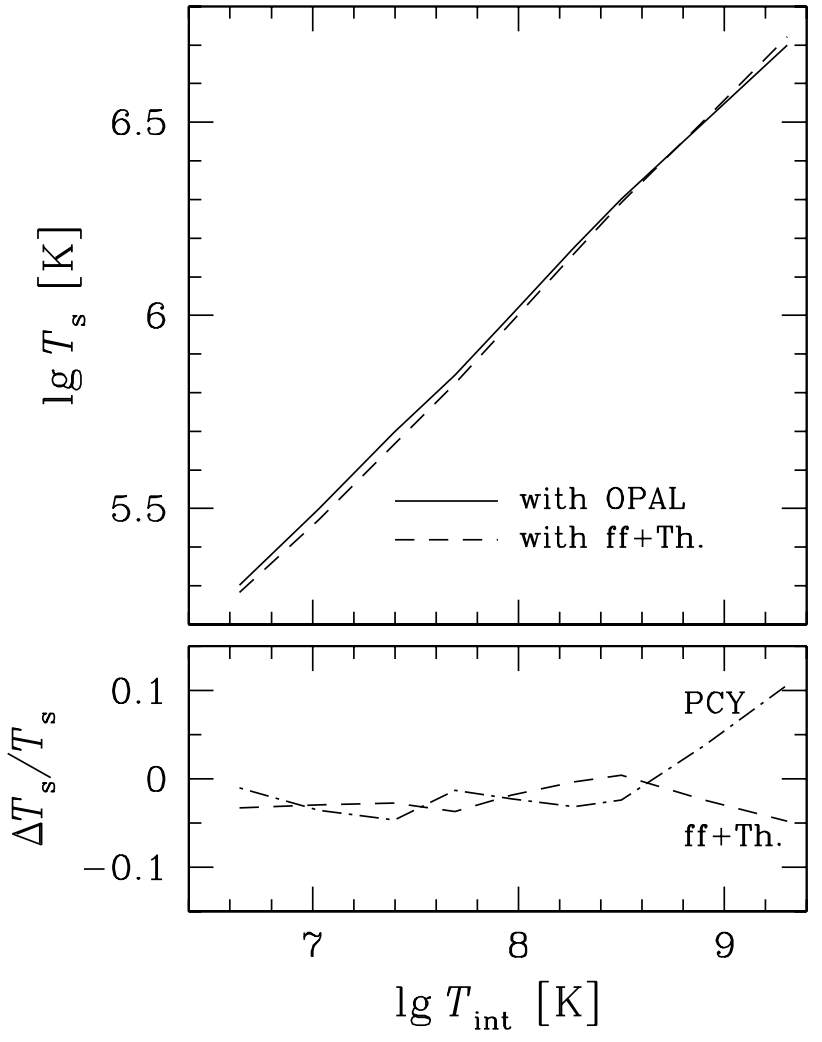

Fig. 1. $T_{\mathrm{s}}\left(T_{\text {int }}\right)$ relations at $B=0$ and $g=2.43 \times 10^{14} \mathrm{~cm} \mathrm{~s}^{-2}$, obtained using OPAL radiative opacities (solid line) and the free-free+Thomson opacities described in the text (dashed line). Lower window: fractional differences between the accurate numerical values of $T_{\mathrm{s}}\left(T_{\mathrm{int}}\right)$ and those obtained using (i) the free-free+Thomson opacity (dashed line) and (ii) the older analytic formula of PCY (dot-dashed line).

curve obtained using Eqs. (14)-(20) and the fit derived in PCY (dot-dashed line) is of similar magnitude. The latter difference arises not only from the fit error (PCY), but also from the improvement of the electron conduction opacity (Potekhin et al. 1999) and, at $T \gtrsim 10^{9} \mathrm{~K}$, from shifting $\rho_{\mathrm{b}}$ to $4 \times 10^{11} \mathrm{~g} \mathrm{~cm}^{-3}$ (for, as noted in PCY, isothermality is not fully reached at $\rho=10^{10} \mathrm{~g} \mathrm{~cm}^{-3}$ for high $T$ ).

Interaction of photons with magnetized plasma depends on their polarization and propagation direction. Accordingly, radiative thermal conductivities and associated opacities along and across the field become different. Rosseland mean opacities $K_{\mathrm{r} \|}$ and $K_{\mathrm{r} \perp}$ were calculated by Silant'ev \& Yakovlev (1980) for various values of $T$ and $B$. We have fitted their numerical results by

$\frac{K_{\mathrm{r}}(\rho, T, 0)}{K_{\mathrm{r} \|}(\rho, T, B)}=1+\frac{A_{1} u+\left(A_{2} u\right)^{2}}{1+A_{3} u^{2}} u^{2}$,

$\frac{K_{\mathrm{r}}(\rho, T, 0)}{K_{\mathrm{r} \perp}(\rho, T, B)}=\frac{1+\left(A_{4} u\right)^{3.5}+\left(A_{5} u\right)^{4}}{1+A_{6} u^{2}}$,

$A_{n}=a_{n}-b_{n} f^{c_{n}}$

where $u=T_{B} /(2 T), K_{\mathrm{r}}(\rho, T, 0)$ is the field-free opacity (19), and the parameter $f$ introduced in Eq. (19) is calculated at $B=0$. The best-fit parameters $a_{n}, b_{n}$ and $c_{n}$ 
Table 1. Coefficients $a_{n}, b_{n}$ and $c_{n}$ of Eq. (23).

\begin{tabular}{lllllll}
\hline$n$ & 1 & 2 & 3 & 4 & 5 & 6 \\
\hline$a_{n}$ & 0.0949 & 0.1619 & 0.2587 & 0.3418 & 0.4760 & 0.2533 \\
$b_{n}$ & 0.0610 & 0.1400 & 0.1941 & 0.0415 & 0.3115 & 0.1547 \\
$c_{n}$ & 0.090 & 0.0993 & 0.0533 & 2.15 & 0.2377 & 0.231 \\
\hline
\end{tabular}

given in Table 1 ensure an average fit error of $5.5 \%$ with the maximum error of $11 \%$.

Asymptotic behaviour of Eqs. (21) and (22) at $u \rightarrow \infty$ agrees with theoretical results of Silant'ev \& Yakovlev (1980): $K_{\mathrm{r} \|}(\rho, T, B)=2 K_{\mathrm{r} \perp}(\rho, T, B)=(\pi / u)^{2} K_{\mathrm{r}}(\rho, T, 0)$ at $f=0$ (Thomson scattering); $K_{\mathrm{r} \|}(\rho, T, B)=$ $K_{\mathrm{r} \perp}(\rho, T, B)$ at $f=1$ (free-free transitions); and $K_{\mathrm{r}} \propto$ $u^{-2}$ at any $f$.

At finite but large $u$ the radiative opacities of fully ionized matter are strongly reduced. The reduction is $\sim 10$ times stronger for scattering than for free-free transitions. Inserting this factor 10 into Eq. (16) and taking into account that, at $B \gtrsim 10^{11} \mathrm{G}$, the NS radiative surface is pushed to $\rho_{\mathrm{s}} \sim B_{12} \mathrm{~g} \mathrm{~cm}^{-3}$ (Ventura \& Potekhin 2001 , one can see that in deep, strongly magnetized photospheric layers Thomson scattering dominates the opacity only at $T_{6} \gtrsim 10 \rho^{2 / 7} \gtrsim 10 B_{12}^{2 / 7}$.

We note that the Rosseland mean opacity due to scattering by free ions, $K_{\mathrm{r}}^{(\mathrm{s}, \mathrm{i})}$, can be obtained from that for electrons, $K_{\mathrm{T}}$, by the simple scaling:

$K_{\mathrm{r}}^{(\mathrm{s}, \mathrm{i})}(\rho, T, B) \approx Z^{3}\left(\frac{m_{\mathrm{e}}}{A m_{\mathrm{u}}}\right)^{2} K_{\mathrm{T}}\left(\rho, T, \frac{Z m_{\mathrm{e}}}{A m_{\mathrm{u}}} B\right)$.

On the contrary, there is no simple scaling for the opacity of photons due to electron-ion (electron bremsstrahlung) and ion-ion (ion bremsstrahlung) collisions. For instance, the processes are well known to be drastically different in the electric dipole approximation.

From the asymptote of $K_{\mathrm{T}}$ at large $u$ we see that the Thomson opacity of ions is $\sim Z$ times larger than that of electrons, if the ions are strongly quantized into Landau levels, which occurs at $T_{6} \ll 0.07(Z / A) B_{12}$. Since the main contribution into the radiative opacity at sufficiently low temperature comes from free-free processes (see above), we can conclude that the Thomson ion scattering cannot contribute appreciably to the Rosseland mean opacity of the photosphere unless $B_{12} \gg 10^{3}(A / Z)^{1.4}$.

\subsubsection{Electron conductivities}

Electron thermal and electrical conduction is the most important process that determines thermal structure and magnetic evolution of NSs. In dense, strongly coupled Coulomb plasmas, typical of NS envelopes, the electron thermal conductivity is mainly determined by electron scattering off ions. At $B=0$ it can be written as

$\kappa=\frac{\pi^{2} k_{\mathrm{B}}^{2} T n_{\mathrm{e}} \tau_{\kappa}}{3 m_{\mathrm{e}}^{*}}$ where $m_{\mathrm{e}}^{*} \equiv m_{\mathrm{e}} \sqrt{1+x_{\mathrm{r}}^{2}}$ is the kinematic electron mass and $\tau_{\kappa}$ is the effective relaxation time.

Recently, Baiko et al. (1998) considerably improved the treatment of $\kappa$ by taking into account multiphonon absorption and emission processes in Coulomb crystals and incipient quasiordering in a Coulomb liquid of ions. Using these results, Potekhin et al. (1999) constructed an effective scattering potential which allowed them to calculate the non-magnetic conductivity in the relaxation time approximation in an analytic form; these analytic expressions described accurately numerical results obtained beyond the framework of the relaxation time approximation. The effective potential has been used then at arbitrary magnetic fields in order to derive practical expressions for evaluation of electrical and thermal conductivities of degenerate electrons in magnetized outer envelopes of NSs (Potekhin 1999). Unlike previous treatments of the electrical conductivities perpendicular to the quantizing magnetic fields (Kaminker \& Yakovlev 1981; Hernquist 1984; Schaaf 1988), Potekhin (1999) went beyond the assumption that $\omega_{\mathrm{g}} \tau_{\kappa} \gg 1$ by introducing an interpolation from the case $\omega_{\mathrm{g}} \tau_{\kappa} \gg 1$ to $\omega_{\mathrm{g}} \tau_{\kappa} \ll 1$. All tensor components of the kinetic coefficients in magnetic fields have been calculated and fitted by analytic formulae; the corresponding Fortran code is available electronically at http://www.ioffe.rssi.ru/astro/conduct/. This code is used here to calculate the temperature profile in the outer envelope of a NS.

In the inner envelope, we need to know the thermal conductivity $\kappa$ only in young NSs, $t \lesssim 100 \mathrm{yr}$, as long as the internal region $\left(r<R_{\mathrm{b}}\right)$ remains non-isothermal (GYP). For this purpose, we will use the non-magnetic conductivities presented by GYP. They generalize the expressions by Potekhin et al. (1999) in two respects. First, the atomic nuclei in the inner envelope cannot be considered as pointlike: the size and shape of nuclear charge distribution significantly affect the conductivity. Second, the electron-phonon scattering in the inner crust at temperatures much below $10^{8} \mathrm{~K}$ changes its character: the so called umklapp processes cease to dominate and the normal processes (with electron momentum transfer within one Brillouin zone) become more important.

The internal temperature in young NSs is relatively high, $T \gtrsim 10^{8} \mathrm{~K}$. Our neglect of the effects of the magnetic fields in the internal region is strictly justified for the case of small magnetization parameter, $\omega_{\mathrm{g}} \tau_{\kappa}<1$. This parameter is shown in Fig. 2 as a function of $T$ for three values of density: near the top and the bottom of the inner envelope (solid lines) and in the middle of the outer envelope (dashed line). We see that the use of non-magnetic $\kappa$ is justified for the NSs with the magnetic fields $B \lesssim 10^{13} \mathrm{G}$ in the inner envelope. However it cannot be justified for higher $B$ in the inner envelope or for $B \gtrsim 10^{10} \mathrm{G}$ in the outer envelope. In such cases the conductivity across the field is suppressed by the large factor $\left[1+\left(\omega_{\mathrm{g}} \tau_{\kappa}\right)^{2}\right]$ and the Hall thermal conductivity may become important. We neglect these effects in the inner envelope. Therefore, our simulations (Sect. 4 ) of cooling of young NSs $(t \lesssim 100 \mathrm{yr})$ 


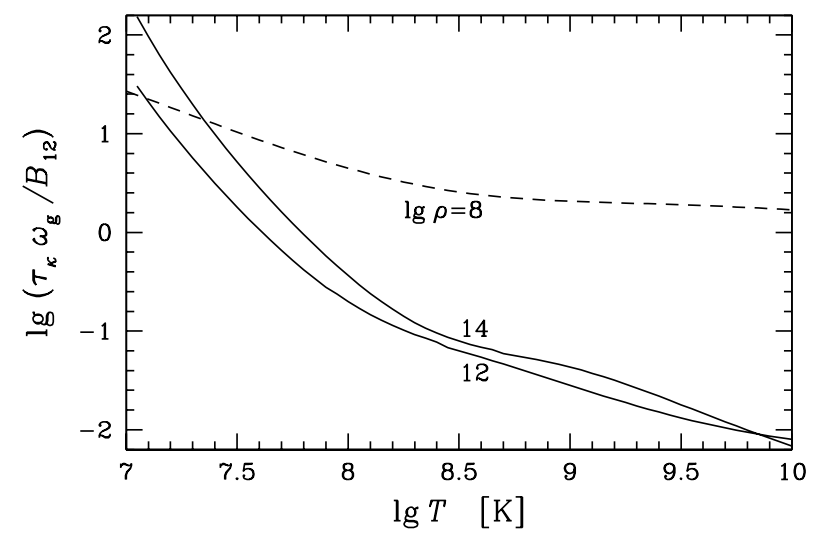

Fig. 2. Electron magnetization parameter at the top and bottom of the inner crust (solid lines) and in the outer envelope (dashed line). The curves are marked with the values of $\log \rho$ $\left[\mathrm{g} \mathrm{cm}^{-3}\right]$.

are justified for $B \lesssim 10^{13} \mathrm{G}$ in the inner envelopes. The results for older NSs, for which the inner envelope is isothermal, are valid at larger $B$ as well.

\section{Thermal structure}

\subsection{Temperature profiles}

We have solved Eq. (7) using the numerical algorithm described in PCY with the EOS and opacities presented in the previous section. We have varied the magnetic field inclination angle $\theta$ from 0 to $90^{\circ}$, the effective temperature $T_{\mathrm{S}}$ from $2 \times 10^{5} \mathrm{~K}$ to $10^{7} \mathrm{~K}$, and the field strength $B$ from 0 to $10^{16} \mathrm{G}$. As clear from the discussion in Sect. 2, our model EOS and opacities may be crude at $B \gtrsim 10^{14} \mathrm{G}$. Further improvements of physics input are required for obtaining more reliable results at such $B$.

Figures 3 and 4 present the calculated $T(\rho)$ profiles for a NS of mass $M=1.4 M_{\odot}$ and radius $R=10 \mathrm{~km}$ $\left(g=2.43 \times 10^{14} \mathrm{~cm} \mathrm{~s}^{-2}\right.$ and $\left.r_{\mathrm{g}} / R=0.413\right)$. The curves in Fig. 3 are calculated at fixed $T_{\mathrm{s}}=10^{6} \mathrm{~K}$. The solid curves show the results of accurate calculations for the magnetic field normal and tangential to the surface (cases of parallel and transverse conduction, Sect. 2.1.2). The dashed lines are obtained for the parallel conduction using the classical thermal conductivity (neglecting the Landau quantization). This approximation becomes inaccurate with increasing $B$. The dot-and-dash lines are calculated for the tangential magnetic field using the transverse conductivity in the limit of $\omega_{\mathrm{g}} \tau_{\kappa} \gg 1$, employed in previous papers (e.g., Hernquist 1984, 1985; Schaaf 1988, 1990a, 1990b; Heyl \& Hernquist 1998a, 1998b). This approximation is inaccurate at lower $B$; a more accurate approximation (solid line) is given by the interpolation (Potekhin 1999) mentioned in Sect. 2.3.2.

Figure 4 shows temperature profiles at four field strengths $B$ and five inclinations $\theta$ for $T_{\text {int }}=10^{8}$ and $10^{9} \mathrm{~K}$ (we do not present the curves for $T_{\text {int }}=10^{8}$ at $B=10^{15} \mathrm{G}$ because the temperature $T(\rho)$ is too small near the surface in the transverse conduction case). The
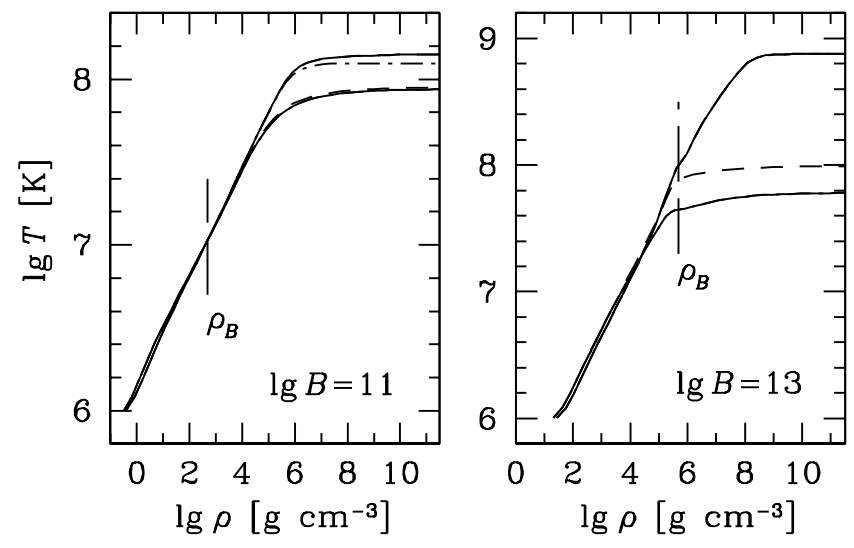

Fig. 3. Temperature profiles through an iron envelope of a NS with $M=1.4 M_{\odot}$ and $R=10 \mathrm{~km}$ for the magnetic field $B=10^{11} \mathrm{G}$ (left panel) and $10^{13} \mathrm{G}$ (right panel) at the fixed effective surface temperature $T_{\mathrm{s}}=10^{6} \mathrm{~K}$. Lower solid curves correspond to $\theta=0$ and upper ones to $\theta=90^{\circ}$. Dashed lines are calculated with the classical thermal conductivity for $\theta=0$, while dot-dashed ones are calculated for $\theta=90^{\circ}$ assuming $\omega_{\mathrm{g}} \tau_{\kappa} \gg 1$ which is traditional for the transverse conduction.

curves show pronounced $\theta$-dependence at high densities starting from the turning point (Sect. 2.3).

The endpoints of the curves in Figs. 3 and 4 lie at the radiative surface $\rho=\rho_{\mathrm{s}}$, where $T=T_{\mathrm{s}}$, and near the neutron drip point. Their behaviour qualitatively agrees with the results of the approximate analytic study of Ventura \& Potekhin (2001). For instance, we confirm the approximate linear dependence $\rho_{\mathrm{s}} \propto B$ and the shift of the turning point to higher densities with increasing $B$. The density $\rho_{\mathrm{B}}$ marked on the graphs is defined by Eq. (1). It separates the strongly- and weakly-quantizing field regimes. We see that the increase of $T$ at $\rho>\rho_{\mathrm{B}}$ (neglected by Heyl \& Hernquist 1998a) can be significant.

The higher the temperature, the wider is the density region, where $\kappa_{\|}$and $\kappa_{\perp}$ are of similar magnitude. Therefore the dependence of the profiles on the inclination $\theta$ and magnetic field strength $B$ is less pronounced at higher $T_{\text {int }}$ showing convergence to the $B=0$ case.

\subsection{Relation between internal and effective temperatures}

A simple analytic fit to $T_{\mathrm{s}}\left(T_{\text {int }}\right)$ was found by Gudmundsson et al. (1983) for iron envelopes at $T_{\mathrm{S}}>$ $3 \times 10^{5} \mathrm{~K}$. Later, using an updated physics input, PCY constructed a more general fit for NSs with (accreted) envelopes containing layers of different chemical elements. In all cases, the scaling law $T_{\mathrm{s}} \propto g^{1 / 4}$ has been confirmed. This approximate law, first noted by Gudmundsson et al. (1983), follows from the thermal structure Eq. (7), if one takes into account that the largest contribution to the integral of this equation comes from the sensitivity strip, being almost independent of the outer boundary condition (cf. Ventura \& Potekhin 2001). The scaling $T_{\mathrm{s}} \propto g^{1 / 4}$ holds also in our case of magnetized envelopes. Therefore 

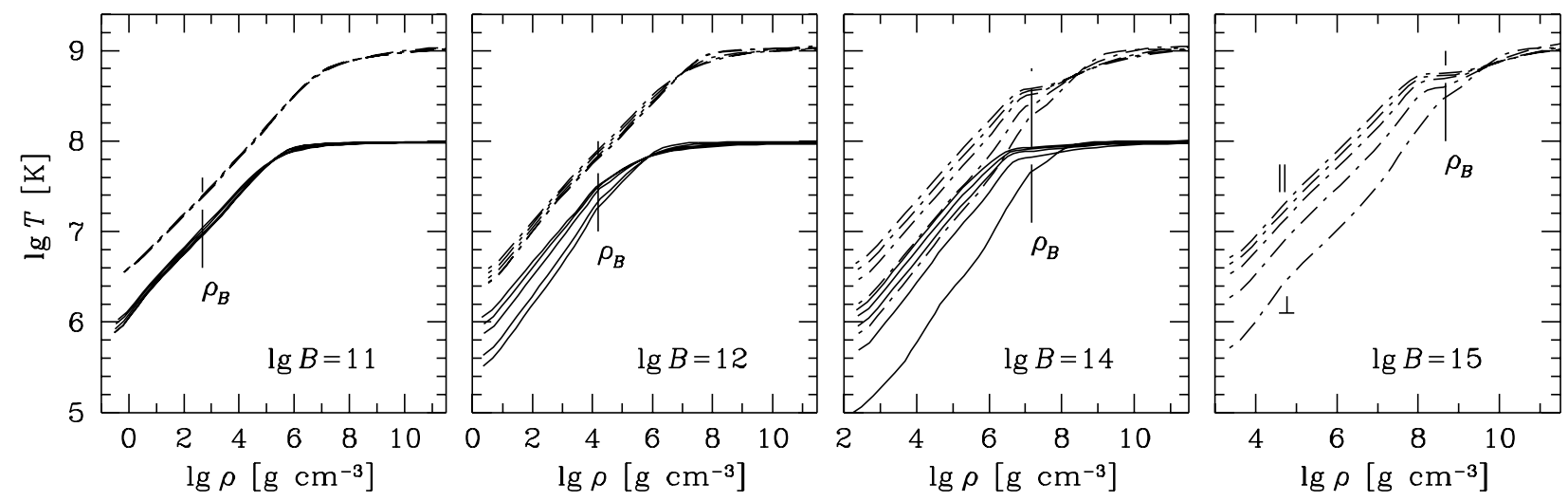

Fig. 4. Temperature profiles through an iron envelope of a NS with $M=1.4 M_{\odot}$ and $R=10 \mathrm{~km}$ at (from left to right) $B=10^{11}$, $10^{12}, 10^{14}$, and $10^{15} \mathrm{G}$. The internal temperature is fixed to $T_{\mathrm{int}}=10^{8} \mathrm{~K}$ (solid lines) or $10^{9} \mathrm{~K}$ (dot-dashed lines). The lines of each group correspond to $\cos \theta=1$ (the lowest line), $0.7,0.4,0.1$, and 0 (the highest line).

the ratio $\mathcal{X}$ of the surface temperature $T_{\mathrm{s}}\left(B, \theta, g, T_{\text {int }}\right)$ at a given magnetic field to the value $T_{\mathrm{s}}^{(0)}\left(g, T_{\mathrm{int}}\right)$ in the absence of the field is practically independent of $g$ :

$T_{\mathrm{s}}\left(B, \theta, g, T_{\mathrm{int}}\right) \approx T_{\mathrm{s}}^{(0)}\left(g, T_{\mathrm{int}}\right) \mathcal{X}\left(B, \theta, T_{\mathrm{int}}\right)$.

Since our outer envelope consists of iron, $T_{\mathrm{S}}^{(0)}$ is given by Eq. (A7) of PCY:

$T_{\mathrm{s}}^{(0)} \approx 10^{6} g_{14}^{1 / 4}\left[(7 \zeta)^{2.25}+(\zeta / 3)^{1.25}\right]^{1 / 4} \mathrm{~K}$,

where $\zeta \equiv T_{\text {int }, 9}-0.001 g_{14}^{1 / 4} \sqrt{7 T_{\text {int }, 9}}, \quad T_{\text {int }, 9} \equiv$ $T_{\text {int }} /\left(10^{9} \mathrm{~K}\right)$ and $g_{14} \equiv g / 10^{14} \mathrm{~cm} \mathrm{~s}^{-2}$.

For the parallel and transverse conduction cases (at the magnetic pole and equator) we have, respectively,

$\mathcal{X}_{\|}\left(B, T_{\mathrm{int}}\right) \approx 1+0.0492 B_{12}^{0.292} / T_{\mathrm{int}, 9}^{0.240}$,

$\mathcal{X}_{\perp}\left(B, T_{\mathrm{int}}\right) \approx \frac{\sqrt{1+0.1076 B_{12}\left(0.03+T_{\mathrm{int}, 9}\right)^{-0.559}}}{\left[1+0.819 B_{12} /\left(0.03+T_{\mathrm{int}, 9}\right)\right]^{0.6463}}$.

These fits have been checked for $B \lesssim 10^{16} \mathrm{G}$ and $10^{7} \mathrm{~K} \leq$ $T_{\text {int }} \leq 10^{9.5} \mathrm{~K}$ with an additional constraint $T_{\mathrm{s}}>2 \times$ $10^{5} \mathrm{~K}$. Maximum residuals ( $11 \%$ and $5 \%$, respectively) occur at the largest $B$.

The effect of the magnetic field on the effective temperature and the quality of the present fit are illustrated in Fig. 5. The magnetic field normal to the surface enhances the photon luminosity, since heat is transported along the field lines $(\|)$. Accordingly, $T_{\mathrm{s}}$ grows up with increasing $B$. In contrast, the tangential magnetic field significantly decreases $T_{\mathrm{s}}$ since the heat propagates across the field lines $(\perp)$. Our results are in qualitative agreement with earlier results of Schaaf (1990a) and Heyl \& Hernquist (1998b), also shown in Fig. 5, although there are quantitative differences. The origin of the large difference from Schaaf (1990a) is not clear, whereas the difference from the results of Heyl \& Hernquist (1998b) can be attributed to our more accurate treatment of electron thermal conductivity, particularly in the nondegenerate region of the envelope. Note that the results of Heyl \& Hernquist (1998b) for parallel conduction do not converge to the $B=0$ results in the limit of high $T_{\mathrm{int}}$. In addition to the above authors, the case of parallel conduction was considered thoroughly by Van Riper (1988) who however did not present any tables or fit expressions for practical applications. We have compared our results with those presented in his Fig. 29. In a hot NS $\left(T_{\text {int }} \gtrsim 3 \times 10^{9} \mathrm{~K}\right)$ his results are in good agreement with ours and satisfy the criterion of convergence to the $B=0$ case. For $B=0$ the agreement is also quite good at any $T_{\text {int }} \gtrsim 10^{7} \mathrm{~K}\left(T_{\mathrm{s}} \gtrsim 3 \times 10^{5} \mathrm{~K}\right)$ but for $B \gtrsim 10^{13} \mathrm{G}$ and $T_{\text {int }} \ll 3 \times 10^{9} \mathrm{~K}$ the magnetized envelopes of Van Riper appear to be much more heat transparent than ours (in contrast, the envelopes of Heyl \& Hernquist 1998b are less transparent than ours at the same conditions). The nature of this difference is also unclear.

\subsection{Variation of temperature over stellar surface}

The dependence of $T_{\mathrm{s}}$ on the angle $\theta$ between the magnetic field and the normal to the surface is most easily described by the model of Greenstein \& Hartke (1983), which implies a superposition of "longitudinal" and "transverse" heat fluxes: $T_{\mathrm{s}}^{4}(\theta)=T_{\mathrm{s}}^{4}(0) \cos ^{2} \theta+T_{\mathrm{s}}^{4}\left(90^{\circ}\right) \sin ^{2} \theta$. This approximation has been used, e.g., by Page (1995); Shibanov \& Yakovlev (1996); Heyl \& Hernquist (1998a). Our numerical calculations confirm that it reproduces accurately (within $\approx 30 \%$ ) the $T_{\mathrm{s}}(\theta)$ dependence. However, a replacement of the power index 4 with 4.5 yields better accuracy (within 10\%):

$$
\begin{aligned}
\mathcal{X}\left(B, \theta, T_{\text {int }}\right)= & {\left[\mathcal{X}_{\|}^{9 / 2}\left(B, T_{\text {int }}\right) \cos ^{2} \theta\right.} \\
& \left.+\mathcal{X}_{\perp}^{9 / 2}\left(B, T_{\text {int }}\right) \sin ^{2} \theta\right]^{2 / 9}
\end{aligned}
$$

Still better accuracy can be reached by varying the power index between 4 and 5 as a function of $B$ and $T_{\text {int }}$.

Figure 6 shows the distribution of the effective temperature from the magnetic pole to the equator. Heavy dots show the results of numerical integration of $T(\rho)$ profiles. Solid lines are obtained using our fit, Eqs. (26)-(30), while short-dashed lines correspond to the approximation of Greenstein-Hartke (1983). For comparison, dot-dashed 

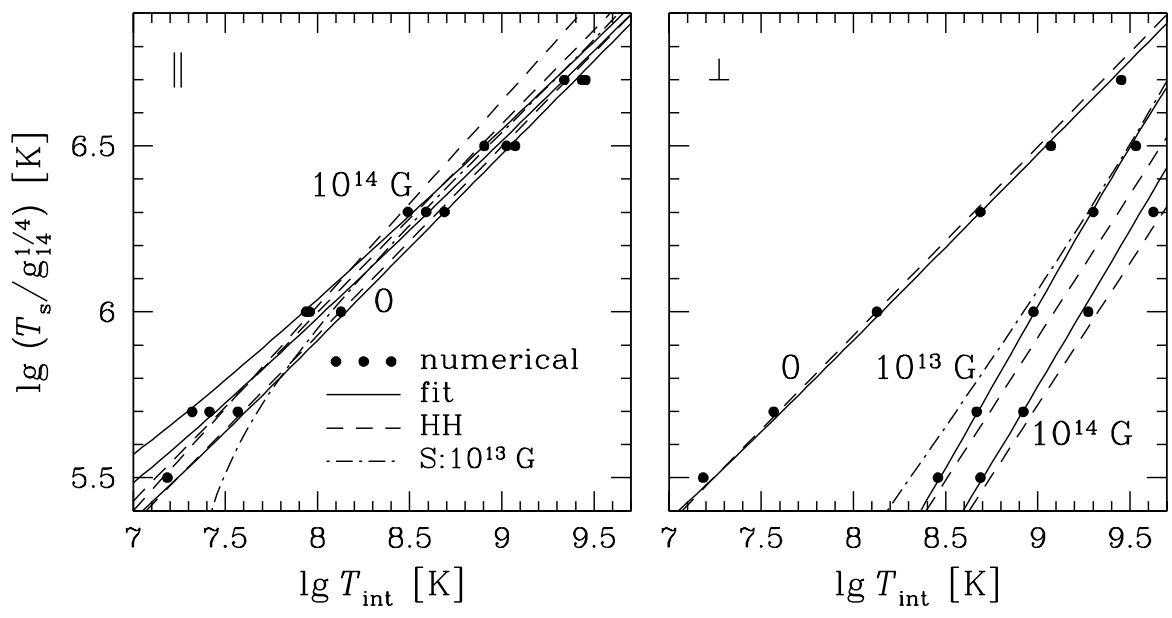

Fig. 5. Dependence of the surface temperature $T_{\mathrm{s}}$ on the internal temperature $T_{\text {int }}$ at the magnetic fields $B=0$, $10^{13}$, and $10^{14} \mathrm{G}$ normal (left panel) or tangential (right panel) to the surface. Numerical data (heavy dots) are compared with the present fit (26)-(29) (solid lines) and with the earlier fits of Schaaf (1990a) (dot-dashed line, for $B=$ $10^{13} \mathrm{G}$ ) and Heyl \& Hernquist (1998b) (dashed lines). lines on the left panel represent the fitting formulae of Schaaf (1990a, 1990b).

Horizontal long-dashed lines in Fig. 6 show $T_{\mathrm{s}}$ which would have been observed at the same $T_{\text {int }}$ if the magnetic field were absent. In qualitative agreement with the results of earlier works (Sect. 1), the thermal flux emergent from the NS interior is suppressed by the magnetic fields in the equatorial region and enhanced near the pole. For a rotating NS, such distribution leads to a pulsating light curve (e.g., Page 1995), which may be observed, for instance, with Chandra in soft X-rays (where the spectral flux of the thermal NS radiation has maximum) or with the $H S T$ in UV.

\subsection{Total photon luminosities}

In order to calculate the cooling curves, one has to know the total NS photon luminosity $L$ at any given $T_{\text {int }}$. In the non-magnetic case, the radiation flux emergent from the surface is given by $F=L /\left(4 \pi R^{2}\right)=\sigma T_{\mathrm{s}}^{4}$, where $T_{\mathrm{s}}=$ $T_{\mathrm{s}}^{(0)}\left(g, T_{\mathrm{int}}\right)$ is known from Eq. (27). In the magnetic case, $F$ should be averaged over the surface. Using the fitting formulae (28)-(30), we have performed such averaging for the dipole configuration of the field (Eq. (10)) at $B_{\mathrm{p}} \leq$ $10^{16} \mathrm{G}, T_{\text {int }}=\left(10^{7}-10^{9.5}\right) \mathrm{K}$, and $r_{\mathrm{g}} / R \leq 0.7$. The ratio of magnetic to non-magnetic fluxes has can be fitted as

$\frac{F(B)}{F(0)}=\frac{1+a_{1} \beta^{2}+a_{2} \beta^{3}+0.007 a_{3} \beta^{4}}{1+a_{3} \beta^{2}}$,

where

$$
\begin{aligned}
\beta & =0.074 \sqrt{B_{12}} T_{\mathrm{int}, 9}^{-0.45}, \\
a_{1} & =\frac{5059 T_{\mathrm{int}, 9}^{3 / 4}}{\left(1+20.4 T_{\mathrm{int}, 9}^{1 / 2}+138 T_{\mathrm{int}, 9}^{3 / 2}+1102 T_{\mathrm{int}, 9}^{2}\right)^{1 / 2}}, \\
a_{2} & =\frac{1484 T_{\mathrm{int}, 9}^{3 / 4}}{\left(1+90 T_{\mathrm{int}, 9}^{3 / 2}+125 T_{\mathrm{int}, 9}^{2}\right)^{1 / 2}}, \\
a_{3} & =\frac{5530\left(1-0.4 r_{\mathrm{g}} / R\right)^{-1 / 2} T_{\mathrm{int}, 9}^{3 / 4}}{\left(1+8.16 T_{\mathrm{int}, 9}^{1 / 2}+107.8 T_{\mathrm{int}, 9}^{3 / 2}+560 T_{\mathrm{int}, 9}^{2}\right)^{1 / 2}},
\end{aligned}
$$

and $B_{12}$ relates to the magnetic pole. The maximum error of this fit is $6.1 \%$ (i.e., $1.5 \%$ for $T_{\mathrm{e}}$ ). Note that Eqs. (28)(30) used for calculating $F(B)$ are actually less accurate by themselves, which lowers the real accuracy of our analytic fits. Nevertheless the latter accuracy seems to be sufficient for cooling simulations.

Note one important feature: the effect of the magnetic field on the $F(B) / F(0)$ ratio becomes weaker with growing $T_{\text {int. }}$. It is explained by the arguments presented in Sect. 3.1. Accordingly, the luminosity of a hot NS cannot be strongly affected even by very high magnetic fields.

\section{Cooling}

Let us discuss briefly the effects of magnetized envelopes on NS cooling. For illustration, we take the models of NSs with two masses, $M=1.3$ and $1.5 M_{\odot}$. For the chosen EOS in the stellar core (Sect. 2), the $1.3 M_{\odot}$ NS has the radius $R=11.86 \mathrm{~km}$. Its central density $\rho_{\mathrm{c}}=1.070 \times 10^{15} \mathrm{~g} \mathrm{~cm}^{-3}$ is lower than the density $\rho_{\mathrm{DU}}=1.298 \times 10^{15} \mathrm{~g} \mathrm{~cm}^{-3}$ at which the powerful direct Urca process of neutrino emission is switched on. Thus the main neutrino emission mechanisms in the NS core are a modified Urca process and neutrino bremsstrahlung in nucleon-nucleon collisions. Accordingly, the $1.3 M_{\odot}$ model gives us the example of slow cooling. For $M=1.5 M_{\odot}$ we have $R=11.38 \mathrm{~km}$ and $\rho_{\mathrm{c}}=1.420 \times 10^{15} \mathrm{~g} \mathrm{~cm}^{-3}>\rho_{\mathrm{DU}}$. The direct Urca process is open in the central kernel of mass $0.065 M_{\odot}$ and radius $2.84 \mathrm{~km}$. This is an example of fast cooling. Further details of these NS models may be found in GYP.

We have calculated the cooling curves of NSs with magnetized envelopes, assuming the dipole magnetic field, Eq. (10). For comparison with earlier papers (Sect. 1), we also consider a familiar model of the field which is radial and constant in magnitude throughout the blanketing envelope. In addition, we have switched on and off superfluidity of neutrons and protons in stellar interiors to demonstrate the combined effects of magnetized envelopes and superfluid interiors on NS cooling. 

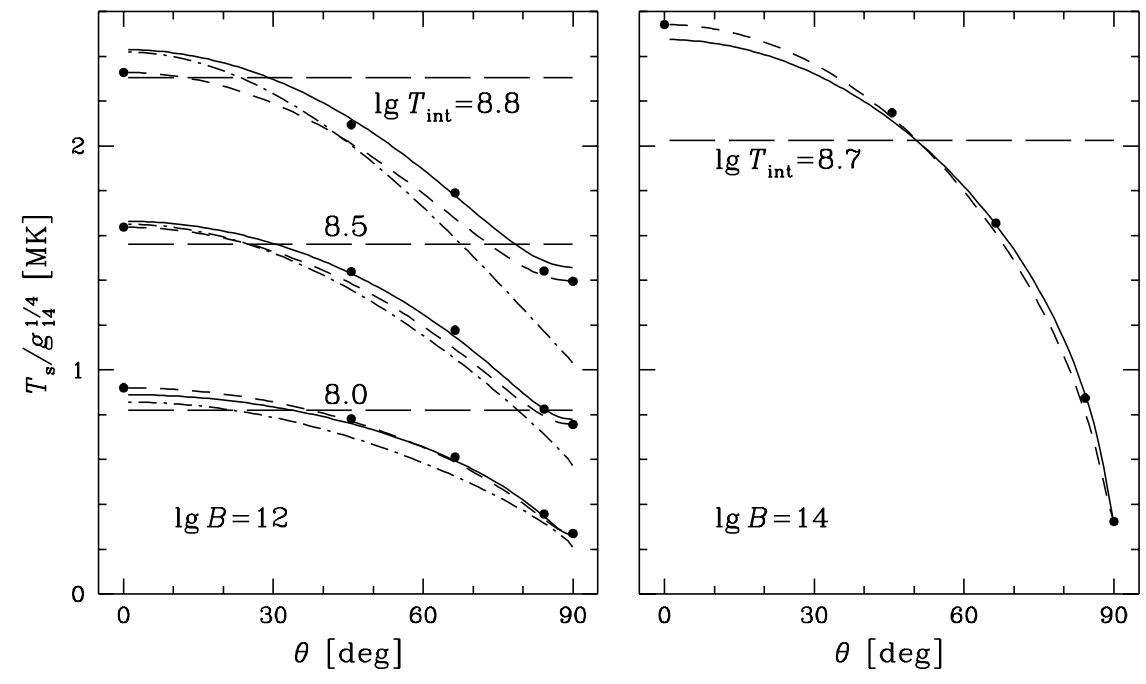

Fig. 6. Dependence of the surface temperature $T_{\mathrm{s}}$ on the inclination angle $\theta$ at constant $B$ and $T_{\text {int }}$. Left panel: $B=10^{12} \mathrm{G}$, $\lg T_{\text {int }}[\mathrm{K}]=8.0,8.5,8.8$; right panel: $B=$ $10^{14} \mathrm{G}, \lg T_{\text {int }}[\mathrm{K}]=8.7$. Dots: numerical results; solid lines: fit; dashed lines: interpolation of Greenstein-Hartke between the accurate values at $\theta=0$ and $90^{\circ}$; long dashes: the case of $B=0$; dot-dashed lines on the left panel: combined fitting formulae of Schaaf (1990a, 1990b).

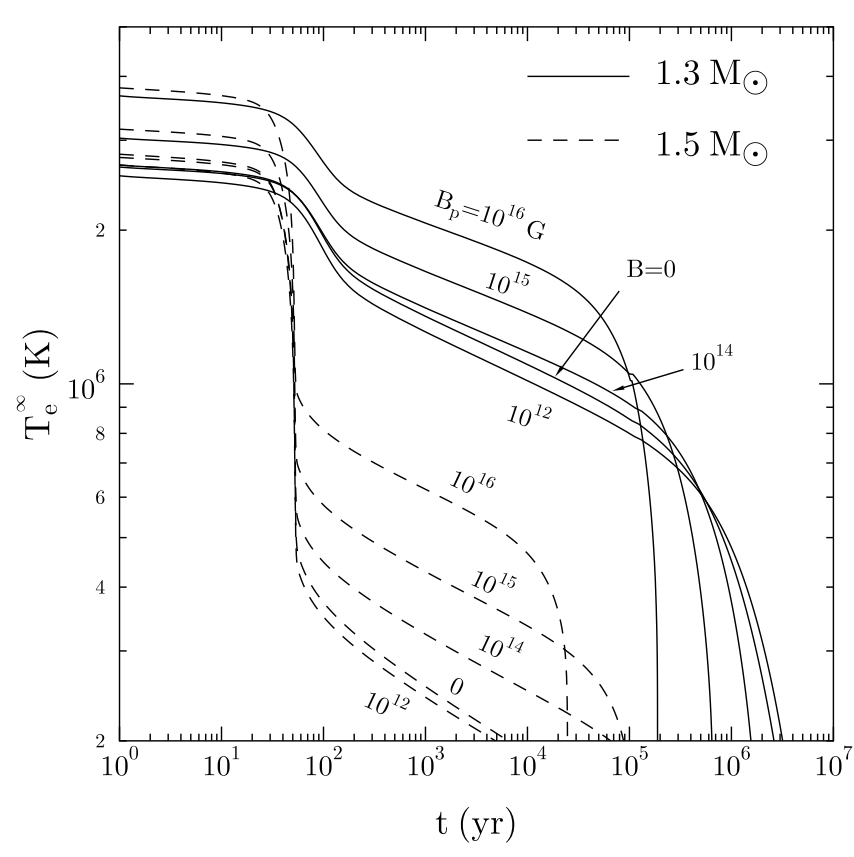

Fig. 7. Cooling curves of non-superfluid $1.3 M_{\odot}$ and $1.5 M_{\odot}$ NSs with dipole magnetic fields of different strengths.

\subsection{Cooling of non-superfluid neutron stars}

\subsubsection{General features}

Figure 7 shows the cooling curves for non-superfluid $1.3 M_{\odot}$ and $1.5 M_{\odot} \mathrm{NSs}$ with dipole magnetic fields. The curves are marked with the magnetic field strengths at the magnetic poles, $B_{\mathrm{p}}$, and display the effective temperature $T_{\mathrm{e}}^{\infty}$, Eq. (9). During the first $50 \mathrm{yr}$ of their lives, $1.3 M_{\odot}$ and $1.5 M_{\odot}$ NSs have nearly the same surface temperatures since the stellar interiors are non-isothermal and thermal evolution of the stellar crust is decoupled from the evolution of the core (e.g., GYP). Later, thermal equilibrium is established in the interior, and the surface temperature of the $1.5 M_{\odot}$ NS becomes much lower due to very powerful direct Urca processes in the stellar kernel.
The results for $B \lesssim 10^{14} \mathrm{G}$ are in good qualitative agreement with those obtained by Page (1995) and Shibanov \& Yakovlev (1996). The thermal state of the stellar interior is almost independent of the magnetic field in the NS envelope at the neutrino cooling stage ( $t \lesssim 10^{4}-10^{5} \mathrm{yr}$ ), but is affected by the magnetic field later, at the photon cooling stage. On the contrary, the surface temperature is always affected by the magnetic field. The dipole field $B \lesssim 10^{13} \mathrm{G}$ makes the blanketing envelope overall less heat-transparent. This lowers $T_{\mathrm{e}}$ at the neutrino cooling stage and slows down cooling at the photon cooling stage. The dipole field $B \gg 10^{13} \mathrm{G}$ makes the blanketing envelope overall more transparent, increasing $T_{\mathrm{e}}$ at the neutrino cooling stage and accelerating the cooling at the photon stage.

Contrary to the case of the dipole magnetic field, any radial field (e.g., Van Riper 1991) would always lower the thermal insulation of the blanketing envelope, increasing $T_{\mathrm{s}}$ at the neutrino cooling stage and accelerating cooling at the photon cooling stage. The radial field would affect the cooling noticeably more than the dipole field.

Even very strong magnetic fields do not change significantly thermal insulation of a hot blanketing envelope (Sect. 3.3). Accordingly, the effects of magnetic fields in a young and hot NSs are not too strong. The strongest effects take place in cold NSs, at the photon cooling stage. Unfortunately, our knowledge of insulating properties of the blanketing envelopes is the poorest for these NSs (Sect. 2). In addition, the magnetic field evolution and reheating processes may become important for these stars, which we ignore for simplicity. Notice that the fields $B \gtrsim 10^{14} \mathrm{G}$ appreciably accelerate the cooling and lower the duration of the neutrino cooling stage.

\subsubsection{Effects of magnetic field and light bending}

Let us consider some effects of the magnetic fields on NS cooling in more detail. Figure 8 demonstrates the effect of dipole or radial magnetic fields on the flux of electromagnetic radiation $F(B)$ detected from a non-superfluid 
1.3 $M_{\odot}$ NS of an age of $25000 \mathrm{yr}$. This is the age of the Vela pulsar (Lyne et al. 1996). This is also a typical age of anomalous X-ray pulsars which are likely to be magnetars (e.g., Mereghetti 2001). Any radial magnetic field is seen to increase the flux. For the field strength $B=4 \times 10^{12} \mathrm{G}$, which can be appropriate for the Vela pulsar, the increase is by a factor of 1.5 , while for ultrahigh $B=10^{15}$ and $10^{16} \mathrm{G}$ the factor is about 4.5 and 9 , respectively.

For the dipole field, however, the detected flux depends on observation direction. The dipole field $B_{\mathrm{p}}=4 \times 10^{12} \mathrm{G}$ reduces the flux averaged over the entire NS surface (solid line) by a factor of 1.3 , while the fields $B_{\mathrm{p}}=10^{15}$ and $10^{16} \mathrm{G}$ amplify it by factors of about 2 and 4 , respectively. The dashed and dotted lines in Fig. 8 refer to the fluxes detected in the direction to the magnetic pole and equator, respectively (assuming the magnetic axis coincides with the rotational one, for simplicity). These lines show the largest difference of the fluxes detected under different angles. They are obtained taking proper account of the general relativity effect of bending of light rays propagating from the NS surface to a distant observer, in the same manner as was done by Page (1995). For comparison, we present also the fluxes calculated neglecting the gravitational ray-bending effect (as if space-time outside the NS were flat). In the absence of light bending effects, the difference of fluxes observed under different angles is quite noticeable. For instance, at $B_{\mathrm{p}}=4 \times 10^{12} \mathrm{G}$ the largest difference would be about $65 \%$. The light bending reduces the largest difference to $14 \%$ for $B_{\mathrm{p}}=4 \times 10^{12} \mathrm{G}$, making it practically negligible. This is explained (e.g., Page 1995) by the fact that gravitational bending of light rays increases the fraction of the NS surface visible by the observer. The observer detects the flux from the larger portion of the surface, that is closer to the flux averaged over observation directions. The light bending effect is strong for our $1.3 M_{\odot}$ and $1.5 M_{\odot}$ NS models. Thus we will neglect weak dependence of fluxes on the detection direction and use the average fluxes and associated effective temperatures $T_{\mathrm{e}}$ in our analysis. Notice that here we mean total (spectral integrated) fluxes but not the fluxes observed in selected spectral bands taking into account interstellar absorption.

Figure 9 shows the effective temperatures of young (1000 yr) non-superfluid $1.3 M_{\odot}$ and $1.5 M_{\odot}$ NSs with dipole and radial magnetic fields of different strengths. The effects of magnetic fields are similar to those in Fig. 8. Since the $1.5 M_{\odot}$ NS undergoes fast cooling, its internal temperature $\left(\sim 10^{7} \mathrm{~K}\right)$ is much lower than in the $1.3 M_{\odot}$ star $\left(\sim 3 \times 10^{8} \mathrm{~K}\right)$. Accordingly, the magnetic fields affect the surface temperature of the $1.5 M_{\odot}$ NS more significantly. Previously, it was suggested (e.g., Heyl \& Hernquist 1997a) that the models of young and hot, slowly cooling NSs with ultramagnetized envelopes could serve as models of anomalous X-ray pulsars and soft gamma repeaters. Although those previous cooling models were rather simplified, they are in qualitative agreement with our more elaborate models. The main outcome of these studies is that even ultrahigh magnetic fields

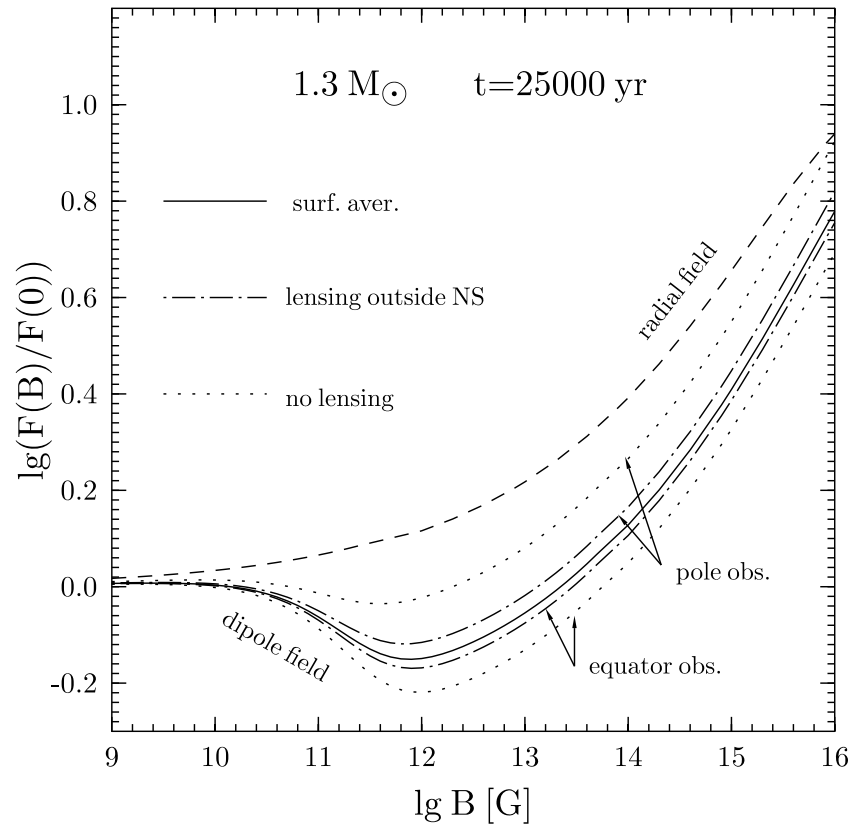

Fig. 8. Effect of dipole or radial surface magnetic fields on the flux of electromagnetic radiation $F(B)$ detected from a nonsuperfluid $1.3 M_{\odot}$ NS of age $t=25000$ yr. Solid line shows the flux averaged over the entire NS surface for the dipole field. Dot-and-dashed lines present the flux observed in the direction towards the magnetic pole (assumed to be aligned with the rotational axis) or towards the magnetic equator. The dotted lines show the same fluxes calculated neglecting gravitational bending of light rays outside the NS.

cannot change appreciably the average surface temperatures of young NSs with iron envelopes (as seen clearly from Figs. 8 and 9). We stress that the local surface temperatures in narrow strips near the magnetic equators appear to be much lower than near the magnetic poles (Sect. 3), but they do not contribute noticeably to the NS luminosity integrated over the surface.

\subsection{Effects of superfluidity and application to the Vela pulsar}

Our analysis shows that the magnetic fields of ordinary pulsars $\left(B \lesssim 10^{13} \mathrm{G}\right)$ do not strongly affect the surface temperature, at least at the neutrino cooling stage. Nevertheless, such fields may influence the theoretical interpretation of observations. For illustration, let us analyse observations of the thermal radiation from the Vela pulsar. We adopt the value of the effective surface temperature $T_{\mathrm{e}}^{\infty}=(6.8 \pm 0.4) \times 10^{5} \mathrm{~K}$ inferred most recently from observations with the Chandra observatory by Pavlov et al. (2001) (at the $1 \sigma$ level) using the hydrogen atmosphere models. This value of $T_{\mathrm{e}}^{\infty}$ is in a good agreement with the value $T_{\mathrm{e}}^{\infty}=(7.85 \pm 0.25) \times 10^{5} \mathrm{~K}$ obtained earlier by Page et al. (1996) from ROSAT observations. Thus we assume the presence of a very thin hydrogen atmosphere (of mass $\lesssim 10^{-13} M_{\odot}$ ) which determines the spectrum of thermal radiation from the NS surface but does not affect 


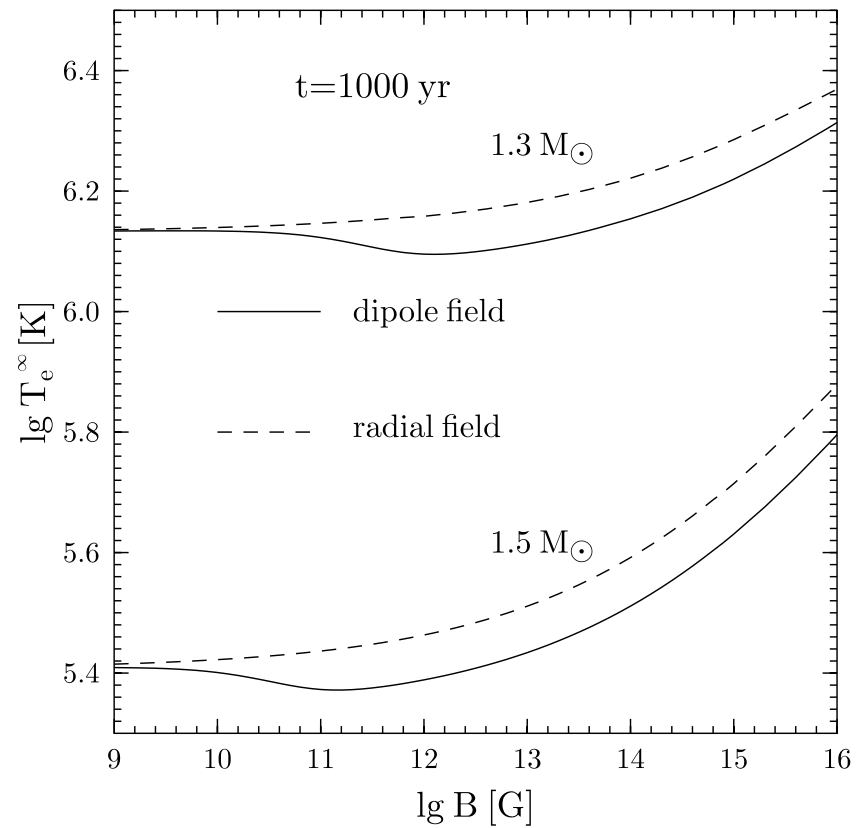

Fig. 9. Effect of dipole or radial surface magnetic fields on the averaged effective surface temperature $T_{\mathrm{e}}^{\infty}$ of non-superfluid $1.3 M_{\odot}$ and $1.5 M_{\odot}$ NSs of age $t=1000 \mathrm{yr}$.

thermal insulation of a blanketing envelope composed mostly of iron. Let us use the slowly cooling $1.3 M_{\odot} \mathrm{NS}$ model for the interpretation of observations. Assuming $B=0$ and no superfluidity in the NS interior, we have, for the age of $25000 \mathrm{yr},{ }^{1} T_{\mathrm{e}}^{\infty} \approx 1.0 \times 10^{6} \mathrm{~K}$, which is higher than the value obtained from observations (Fig. 10). The dipole magnetic field $B_{\mathrm{p}}=4 \times 10^{12} \mathrm{G}$ lowers $T_{\mathrm{e}}^{\infty}$ by $8 \%$ (Fig. 8), not sufficient to attain the required value.

It is well known that neutron or proton superfluidity in the NS core affects the cooling. For illustration, we adopt the same simplified model of nucleon superfluidity which has been used in a number of previous works (Yakovlev et al. 1999 and references therein). In this model, the neutron pairing is assumed to be in the triplet state, while the proton pairing occurs in the singlet state of a nucleonnucleon pair. The critical temperatures $T_{\mathrm{cn}}$ and $T_{\mathrm{cp}}$ of the neutron and proton superfluids are assumed to be constant and treated as free parameters to be adjusted to observational data. Figure 10 demonstrates that taking the neutron and proton superfluidities with certain values of $T_{\mathrm{cn}}$ and $T_{\mathrm{cp}}$ we can lower $T_{\mathrm{e}}$ of our Vela model to the observed values. This can be done for all three models of the envelope, with $B=0$ as well as with the dipole and radial fields. In the given examples, the lowering of $T_{\mathrm{e}}$ for the dipole or field-free cases is produced by neutrino emission due to Cooper pairing of neutrons, while for the radial field the lowering is produced by neutrino emission due to Cooper pairing of neutrons and protons. In these

\footnotetext{
${ }^{1}$ We have verified that $T_{\mathrm{e}}^{\infty}$ is almost independent of the NS mass, for the given EOS, as long as $M$ is lower than the critical value $1.44 M_{\odot}$ at which the direct Urca process becomes open in the stellar interior.
}

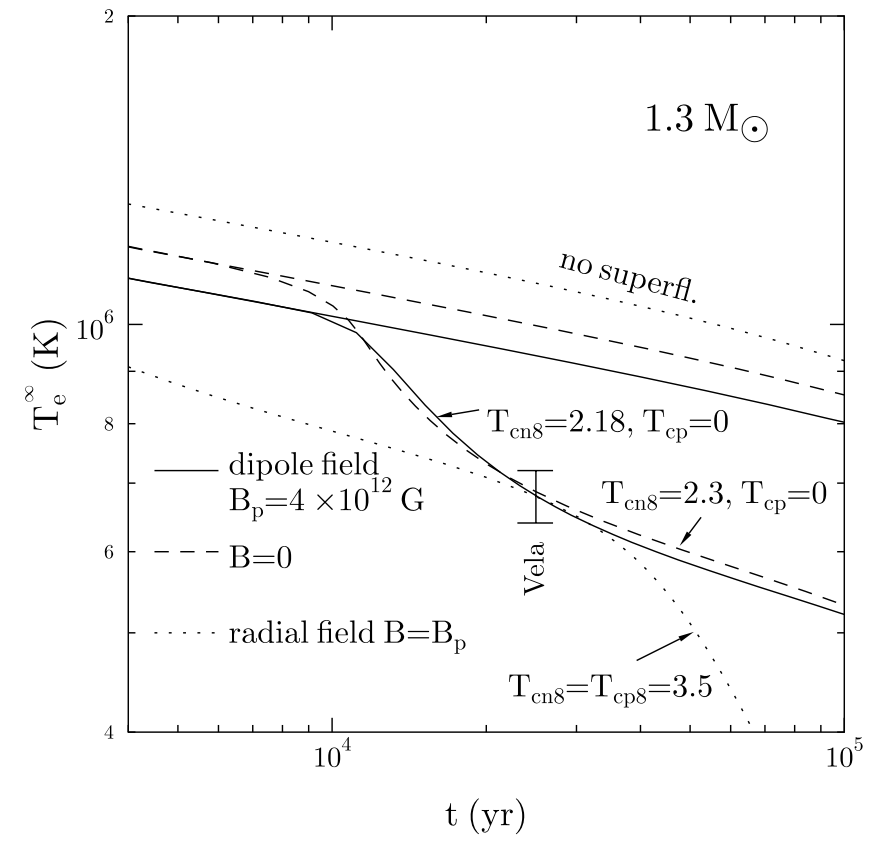

Fig. 10. Cooling of $1.3 M_{\odot}$ NS with $B=0$, with the dipole magnetic field $B_{\mathrm{p}}=4 \times 10^{12} \mathrm{G}$ or with the radial field of the same strength. The upper curves are calculated assuming no superfluidity in the NS interior while the lower ones are calculated for certain critical temperatures of the neutron and proton superfluids in the NS cores (the values of $T_{\mathrm{cn}}$ and $T_{\mathrm{cp}}$ are indicated near the curves in units of $10^{8} \mathrm{~K}$ ). Error bars show the possible interval of the surface temperatures of the Vela pulsar (see text).

three cases we need different critical temperatures $T_{\mathrm{cn}}$ and $T_{\mathrm{cp}}$. In each case the choice of $T_{\mathrm{cn}}$ and $T_{\mathrm{cp}}$ is not unique.

Generally, if we fix the blanketing envelope model, we can determine the domains of $T_{\mathrm{cn}}$ and $T_{\mathrm{cp}}$ values in the $T_{\mathrm{cn}}-T_{\mathrm{cp}}$ plane which force the NS to have $T_{\mathrm{e}}^{\infty}$ within the given errorbar by the specified age $t$. These domains (hatched regions) are shown in Fig. 11 for the Vela pulsar with a non-magnetic envelope, and also for the envelope with dipole and radial magnetic fields.

The domain for the non-magnetic envelope has a complicated X-shape. The dipole magnetic field facilitates a lowering of $T_{\mathrm{e}}$ to the required errorbar. The domain of acceptable values of $T_{\mathrm{cn}}$ and $T_{\mathrm{cp}}$ becomes narrower overall and splits into two parts (Fig. 11). In principle, in these two cases we do not need proton superfluidity to explain the observations (Fig. 10): the neutrino emission due to Cooper pairing of neutrons is sufficiently strong by itself to lower $T_{\mathrm{e}}$. On the other hand, were the magnetic field radial, it would raise $T_{\mathrm{e}}$ and complicate the adjusting of $T_{\mathrm{e}}$ to observations. Neutrino emission due to Cooper pairing of neutrons is insufficient to lower $T_{\mathrm{e}}$, and the emission due to Cooper pairing of protons is needed. The domain acquires a qualitatively different shape: the proton superfluidity cannot be too weak $\left(\log T_{\mathrm{cp}} \gtrsim 8.3\right)$.

The domains of $T_{\mathrm{cn}}$ and $T_{\mathrm{cp}}$ obtained for the Vela pulsar can be combined further with analogous domains determined for other cooling NSs in attempt to impose 


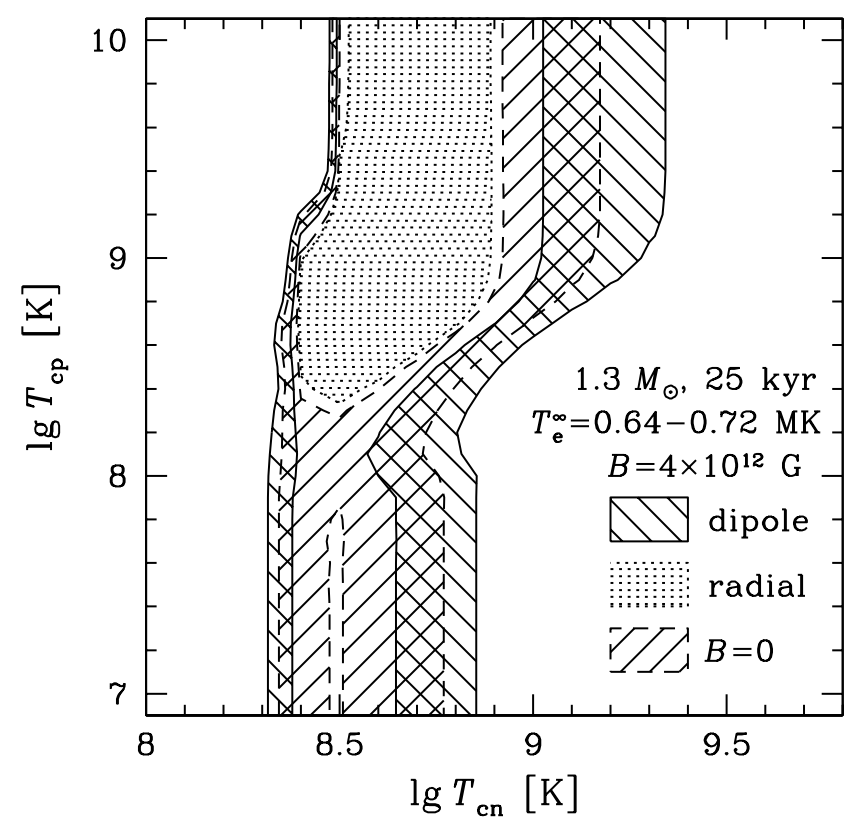

Fig. 11. Domains (hatched) of $T_{\mathrm{cn}}$ and $T_{\mathrm{cp}}$ for which the $1.3 M_{\odot}$ NS with the superfluid core has the surface temperature $T_{\mathrm{e}}^{\infty}=(6.8 \pm 0.4) \times 10^{5} \mathrm{~K}$ by the age of the Vela pulsar. Solid lines enclose the domains for the NS with the dipole magnetic field $\left(B_{\mathrm{p}}=4 \times 10^{12} \mathrm{G}\right)$; dashed lines are for the non-magnetized NS and dots are for the radial field $\left(B=B_{\mathrm{p}}\right)$.

restrictions on the values of $T_{\mathrm{cn}}$ and $T_{\mathrm{cp}}$ in the stellar cores. A preliminary analysis of such a kind has been done by Yakovlev et al. (1999). However, further work remains to be done to constrain strongly the critical temperatures: one should take into account new observational data on thermal radiation from isolated NSs and new theoretical models of cooling NSs with superfluid cores; particularly, one has to incorporate density dependence of $T_{\mathrm{cn}}$ and $T_{\mathrm{cp}}$ over the stellar core. Such work is beyond the scope of the present paper, but our illustrative examples show that proper analysis of the NS superfluidity may require consideration of the effects of the magnetic field on the NS blanketing envelope.

\section{Conclusions}

We have analysed the thermal structure of neutron star envelopes with typical pulsar magnetic fields $10^{11}-10^{13} \mathrm{G}$ and with ultrahigh magnetic fields up to $10^{16} \mathrm{G}$. We have used (Sect. 2) modern data on equation of state and thermal conductivities of magnetized neutron star envelopes. In particular, we have proposed an analytic model of the radiative thermal conductivity limited by the Thomson scattering and free-free absorption of photons in a magnetized plasma. We have used the values of thermal conductivity of degenerate electrons, updated recently by proper treatment of dynamical ion-ion correlations which affect electron-ion scattering. We have calculated the temperature profiles (Sect. 3) in the neutron star envelope for any inclination of the magnetic field to the surface and obtained a fit expression which relates the internal temperature of the neutron star to the local effective surface temperature. We have also calculated and fitted the relation between the internal temperature and total surface luminosity (or effective temperature) for the dipole magnetic fields $B \lesssim 10^{16} \mathrm{G}$.

Furthermore, we have performed (Sect. 4) simulations of cooling of neutron stars with dipole or radial magnetic fields in the envelopes. In agreement with the previous studies of Page (1995) and Shibanov \& Yakovlev (1996), we have found that the effects of the two magnetic field configurations on neutron star cooling are qualitatively different. We have briefly discussed cooling of young and middle-aged neutron stars with ultramagnetized envelopes (magnetars as the models of soft gamma repeaters and anomalous X-ray pulsars) and the effect of surface magnetic fields on constraining critical temperatures of the neutron and proton superfluids in the cores of ordinary pulsars, considering the Vela pulsar as an example.

We stress that our results are less reliable for cold neutron stars $\left(T_{\mathrm{s}} \lesssim 3 \times 10^{5} \mathrm{~K}\right)$ with very strong magnetic fields $B \gtrsim 10^{13} \mathrm{G}$ because of lack of knowledge of ionization state and thermal conductivity of the outermost parts of the cold blanketing envelopes. Further work is required to fill these gaps. In this paper, we have considered the blanketing envelope composed of iron and have not analysed the envelopes containing light elements. The presence of light elements generally reduces thermal insulation of the envelope. This effect has been studied in detail for non-magnetic envelopes (PCY) and is expected to be important for magnetic envelopes as well (Heyl \& Hernquist 1997a). We are planning to address this matter in a future work.

Acknowledgements. We are grateful to G. Pavlov for encouragement and for providing us with the results of observations (Pavlov et al. 2001) before publication, to U. Geppert for stimulating suggestions, to Yu. A. Shibanov and K. P. Levenfish for helpful discussions, and to the referee, Dany Page, for useful remarks. This work was supported by RFBR (grant No. 99-0218099).

\section{References}

Baiko, D. A., \& Yakovlev, D. G. 1999, A\&A, 342, 192

Baiko, D. A., Kaminker, A. D., Potekhin, A. Y., \& Yakovlev, D. G. 1998, Phys. Rev. Lett., 81, 5556

Blandford, R. D., \& Hernquist, L. 1982, J. Phys. C, 15, 6233

Berestetskiǔ, V. B., Lifshitz, E. M., \& Pitaevskiŭ, L. P. 1992, Quantum Electrodynamics (Pergamon, Oxford)

Broderick, A., Prakash, M., \& Lattimer, J. M. 2000, ApJ, 537, 351

Chabrier, G., \& Potekhin, A. Y. 1998, Phys. Rev. E, 58, 4941

Ginzburg, V. L., \& Ozernoy, L. M. 1964, Zh. Eksp. Teor. Fiz., 47, 1030 (Engl. transl.: Sov. Phys. - JETP 20, No. 3)

Gnedin, O. Y., Yakovlev, D. G., \& Potekhin, A. Y. 2001, MNRAS, 324, 725 (GYP)

Greenstein, G., \& Hartke, G. J. 1983, ApJ, 271, 283

Gudmundsson, E. H., Pethick, C. J., \& Epstein, R. I. 1983, ApJ, 272, 286 
Hernquist, L. 1984, ApJS, 56, 325

Hernquist, L. 1985, MNRAS, 213, 313

Heyl, J. S., \& Hernquist, L. 1997a, ApJ, 489, L67

Heyl, J. S., \& Hernquist, L. 1997b, ApJ, 491, L95

Heyl, J. S., \& Hernquist, L. 1998a, MNRAS, 300, 599

Heyl, J. S., \& Hernquist, L. 1998b, preprint [astro-ph/9808126]

Hummer, D. G. 1988, ApJ, 327, 477

Iglesias, C. A., \& Rogers, F. J. 1996, ApJ, 464, 943

Kaminker, A. D., \& Yakovlev, D. G. 1981, Theor. Math. Phys., 49, 1012

Kaminker, A. D., Pethick, C. J., Potekhin, A. Y., Thorsson, V., \& Yakovlev, D. G., 1999, A\&A, 343, 1009

Karzas, W. J., \& Latter, R. 1961, ApJS, 6, 167

Kouveliotou, C., Dieters, S., Strohmayer, T., et al. 1998, Nature, 393, 235

Kouveliotou, C., Strohmayer, T., Hurley, K., et al. 1999, ApJ, 510, L115

Lyne, A. G., Pritchard, R. S., Graham-Smith, F., \& Camilo, F. 1996, Nature, 381, 497

Mereghetti, S. 2001, in The Neutron Star - Black Hole Connection, ed. C. Kouveliotou, J. van Paradijs, \& J. Ventura, NATO ASI Ser. (Kluwer, Dordrecht), in press [astro-ph/9911252]

Negele, J. W., \& Vautherin, D. 1973, Nucl. Phys. A, 207, 298

Nomoto, K., \& Tsuruta, S. 1987, ApJ, 312, 711

Page, D. 1995, ApJ, 442, 273

Page, D. 1997, ApJ, 479, L43

Page, D. 1998, in The Many Faces of Neutron Stars, ed. R. Buccheri, J. van Paradijs, \& M. A. Alpar (Kluwer, Dordrecht), 539

Page, D., \& Applegate, J. H. 1992, ApJ, 394, L17

Page, D., Shibanov, Yu. A., \& Zavlin, V. E. 1996, in Röntgenstrahlung from the Universe, ed. H. U. Zimmermann, J. E. Trümper, \& H. Yorke, MPE Report 263 (MPE, Garching), 173

Pavlov, G. G., Zavlin, V. E., Sanwal, D., Burtwitz, V., \& Garmire, G. P. 2001, ApJ, 552, L129

Pethick, C. J. 1992, Rev. Mod. Phys., 64, 1133

Potekhin, A. Y. 1999, A\&A, 351, 787

Potekhin, A. Y., \& Yakovlev, D. G. 1996, A\&A, 314, 341; erratum 1997, A\&A, 327, 442
Potekhin, A. Y., Baiko, D. A., Haensel, P., \& Yakovlev, D. G. 1999, A\&A, 346, 345

Potekhin, A. Y., Chabrier, G., \& Yakovlev, D. G. 1997, A\&A, 323, 415 (PCY)

Prakash, M., Ainsworth, T. L., \& Lattimer, J. M. 1988, Phys. Rev. Lett., 61, 2518

Rajagopal, M., Romani, R., \& Miller, M. C. 1997, ApJ, 479, 347

Rogers, F. J., Swenson, F. J., \& Iglesias, C. A. 1996, ApJ, 456, 902

Schaaf, M. E. 1988, A\&A, 205, 335

Schaaf, M. E. 1990a, A\&A, 227, 61

Schaaf, M. E. 1990b, A\&A, 235, 499

Shapiro, S. L., \& Teukolsky, S. A. 1983, Black Holes, White Dwarfs, and Neutron Stars, The Physics of Compact Objects (Wiley, New York)

Shibanov, Yu. A., \& Yakovlev, D. G. 1996, A\&A, 309, 171

Shibanov, Yu. A., Potekhin, A. Y., Yakovlev, D. G., \& Zavlin, V. E. 1998, in The Many Faces of Neutron Stars, ed. R. Buccheri, J. van Paradijs, \& M. A. Alpar (Kluwer, Dordrecht), 553

Silant'ev, N. A., \& Yakovlev, D. G. 1980, Ap\&SS, 71, 45

Suh, I.-S., \& Mathews, G. J. 2001, ApJ, 546, 1126

Taylor, J. H., Manchester, R. N., \& Lyne, A. G. 1993, ApJS, 88,529

Thompson, C., \& Duncan, R. C. 1995, MNRAS, 275, 255

Thorne, K. S. 1977, ApJ, 212, 825

Thorolfsson, A., Rögnvaldsson, Ö. E., Yngvason, J., \& Gudmundsson, E. H. 1998, ApJ, 502, 847

Urpin, V., \& Konenkov, D. 1997, MNRAS, 292, 167

Urpin, V. A., \& Shalybkov, D. A. 1995, A\&A, 294, 117

Van Riper, K. 1988, ApJ, 329, 339

Van Riper, K. 1991, ApJS, 75, 449

Ventura, J., \& Potekhin, A. Y. 2001, in The Neutron Star Black Hole Connection, ed. C. Kouveliotou, J. van Paradijs, \& J. Ventura, NATO ASI Ser. (Kluwer, Dordrecht), in press [astro-ph/0104003]

Yakovlev, D. G., \& Kaminker, A. D. 1994, in The Equation of State in Astrophysics, ed. G. Chabrier, \& E. Schatzman (Cambridge University Press), 214

Yakovlev, D. G., Levenfish, K. P., \& Shibanov, Yu. A. 1999, Physics-Uspekhi, 42, 737 